SAND95-0447

Distribution

Unlimited Release

Category UC-400

\title{
Plasma Technology DIRECTORY
}

\author{
Pamela P. Ward \\ Properties of Organic Materials Department \\ and \\ Gay L. Dybwad \\ Electronic Processing Department \\ Sandia National Laboratories \\ Albuquerque, New Mexico 87185
}

March 1, 1995

The Plasma Technology Directory has two main goals:

1. Promote, coordinate, and share plasma technology experience and equipment within the Department of Energy.

2. Facilitate technology transfer to the commercial sector where appropriate.

In addition, we solicit new names and plasma technologies in preparation for a second edition. If you are interested fill out the form provided at the end of this Directory.

The first edition of the Plasma Technology Directory has been compiled in cooperation with the Department of Energy's National Laboratories.

Argonne National Laboratory, Argonne, IL Brookhaven National Laboratory, Upton, NY

Idaho National Engineering Laboratory, Idaho Falls, ID

Lawrence Berkeley Laboratory, Berkeley, CA

Lawrence Livermore Laboratory, Livermore, CA

Los Alamos National Laboratory, Los Alamos, NM

National Renewable Energy Laboratory, Golden, CO

Oak Ridge National Laboratories, Oak Ridge, TN

Pacific Northwest Laboratory, Richland, WA

Sandia National Laboratories, Albuquerque, NM, and Livermore, CA

This work was supported by the United States Department of Energy under Contract DE-AC04-94AL85000. 


\section{Contents}

Section

Page

General Laboratory Information ................................................................................. ii

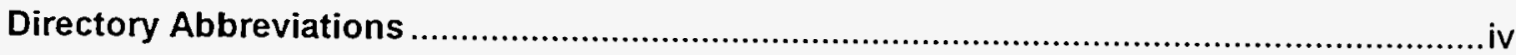

Laboratory Staff (alphabetical list of personnel with plasma expertise) ...........................

\section{Laboratories (personnel arranged by Laboratory)}

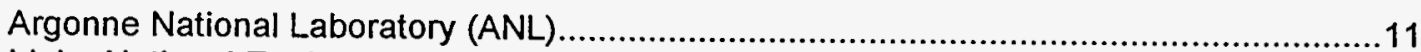

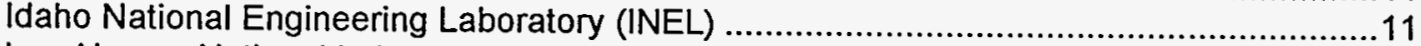

Los Alamos National Laboratory (LANL) ….......................................................... 12

Sandia National Laboratories, California (SNL, CA) ................................................. 13

Sandia National Laboratories, New Mexico (SNL, NM) ............................................. 14

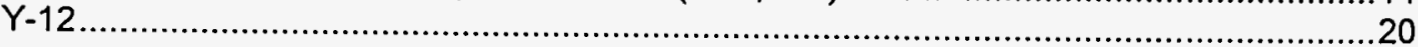

\section{Technologies (personnel arranged by technology area)}

Accelerators

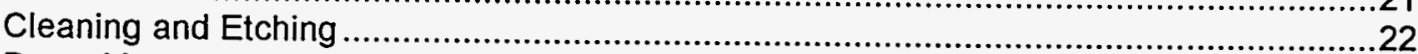

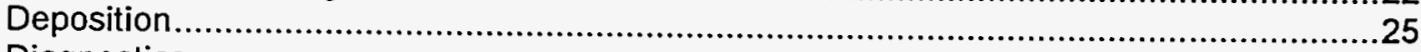

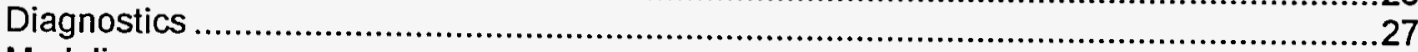

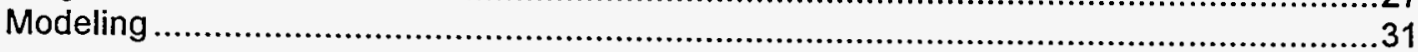

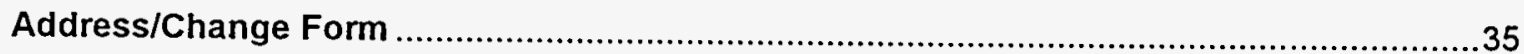

\section{Acknowledgments}

The authors wish to thank Walter P. Schimmel, Industrial Partnership Development, Department 4211 , Sandia National Laboratories, New Mexico, for aiding us in contacting the Technology Transfer department in the ten laboratories.

We also thank Patty Guyer and Judy Crane,12615, for formatting the information for publication.

\section{DISCLAIMER}

This report was prepared as an account of work sponsored by an agency of the United States Government. Neither the United States Government nor any agency thereof, nor any of their employees, makes any warranty, express or implied, or assumes any legal liability or responsibility for the accuracy, completeness, or usefulness of any information, apparatus, product, or process disclosed, or represents that its use would not infringe privately owned rights. Reference herein to any specific commercial product, process, or service by trade name, trademark, manufacturer, or otherwise does not necessarily constitute or imply its endorsement, recommendation, or favoring by the United States Government or any agency thereof. The views and opinions of authors expressed herein do not necessarily state or reflect those of the United States Government or any agency thereof. 


\section{DISCLAIMER}

Portions of this document may be illegible in electronic image products. Images are produced from the best available original document. 


\section{General Laboratory Information}

\section{Laboratory Addresses and Technology Transfer Contacts}

Mr. Steven Lake

Argonne National Laboratory

9700 South Cass Avenue

Argonne, IL 60439

Ms. Margarot Bogosian

(516) $282-7338$

Brookhaven National Laboratory

Building 902C

Upton, NY 11973

Ms. Ann Rydalch

(208) $526-1010$

Idaho National Engineering Laboratory

EG\&G Idaho, Inc.

P.O. Box 1625

Idaho Falls, ID 83415

Mr. Kris Kniel

(708) $252-5685$

Lawrence Berkeley Laboratory

Technology Transfer Department

1 Cyclotron Road, Bldg. 90-1070

Berkeley, CA 94720

Mr. Dave Conrad

(510) $422-7839$

Operations Manager

Lawrence Livermore Laboratory

Technology Transfer Initiatives Program

P.O. Box 80X, L-795

Livermore, CA 94550

Ms. Cheryl Bergor

(505) $665-9090$

Industrial Partnerships

Los Alamos National Lab

P. O. Box 1663, MS K763

Los Alamos, NM 87545

Mr. Kenoall Touryan

National Renewable Energy Laboratory

Technology Transfer Office

1617 Cole Boulevard

Golden, CO 80401-3393

Mr. Marvin Clement

(509) $375-2789$

(510) $426-7020$
Mr. Walter Schimmel

(505) 271-7246

Sandia National Laboratories

P. O. Box 5800

Albuquerque, NM 87185

Mr. Walter Schimmel

Sandia National Laboratories

P. O. Box 969

Livermore, CA 94551

Mr. D. H. Johnson

(615) $574-0868$

Oakridge National Laboratory

Y-12 Technology Transfer Program

P. O. Box 2009

Oak Ridge, TN 37381

Pacific Northwest Laboratory

P.O. Box 999, K1-17

Richland, WA 99352 


\section{Directory Abbreviations}

\section{Laboratories}

$\begin{array}{ll}\text { ANL } & \text { Argonne National Laboratory } \\ \text { INEL } & \text { Idaho National Engineering Laboratory } \\ \text { LANL } & \text { Los Alamos National Laboratory } \\ \text { SNL, CA } & \text { Sandia National Laboratories, California } \\ \text { SNL, NM } & \text { Sandia National Laboratories, New Mexico } \\ \text { Y-12 } & \text { Oak Ridge National Laboratories }\end{array}$

\section{Technical Terms}

$\begin{array}{ll}\text { AES } & \text { auger electron spectroscopy } \\ \text { ECM } & \text { environmentally conscious manufacturing } \\ \text { ECR } & \text { electron cyclotron resonance (plasmas) } \\ \text { EUV } & \text { extreme ultraviolet } \\ \text { FTIR } & \text { fourier transform infrared (spectroscopy) } \\ \text { ICF } & \text { inertial confinement fusion } \\ \text { IRIS } & \text { imaging of radical interactions with surfaces } \\ \text { ISS } & \text { ion scattering spectroscopy } \\ \text { KEV } & \text { kilo-electron volts } \\ \text { LTE } & \text { local thermodynamic equilibrium } \\ \text { MEV } & \text { mega-electron volts } \\ \text { PECVD } & \text { plasma enhanced chemical vapor deposition } \\ \text { PIC } & \text { particle-in-cell } \\ \text { PR } & \text { photoresist } \\ \text { RF } & \text { radio frequency } \\ \text { RIE } & \text { reactive ion etch } \\ \text { SIMS } & \text { secondary ion mass spectrometry } \\ \text { VUV } & \text { vacuum ultraviolet }\end{array}$




\section{Plasma Technology Directory}

\section{Personnel}

\begin{tabular}{lcccc} 
Name & Facility/Dept. & Phone & $\begin{array}{c}\text { Mail } \\
\text { Stop }\end{array}$ & E-Mail Address \\
\hline Apblett, Chris & SNL, NM/1323 & (505) $844-4487$ & & apbletca@sandia.gov
\end{tabular}

Expertise: Electron cyclotron resonance system capable of depositing thin films on 6-inch silicon substrates

Bailey, James E.

SNL, NM/1277

(505) $845-7203$

1196

jebaile@sandia.gov

Expertise: Z-pinch, laser-produced, and ion diode plasmas, plasma spectroscopy, time and space resolved spectra

Barbour, J. Charles

SNL, NM/1111

(505) $844-5517$

1056

jcbarbo@sandia.gov

Expertise: Plasma assisted deposition, ECR accelerations

Bartel, Tim

SNL, NM/1514

(505) 844-0124

0826

tjbarte@cfd.sandia.gov

Expertise: Numerical simulation of plasma etch and deposition reactors and processes by self-consistent particle method

Bastasz, R.

SNL, CA/8347

(510) 294-2013

9162

bastasz@vaxm.gat.com

Expertise: Plasma-surface interactions, SIMS, AES, ISS

Blackwell, Bennie

SNL, NM/1514

(505) 845-8844

0826

blblack@sandia.gov

Expertise: Modeling of thermal conduction, convection and radiation process to design and improve plasma equipment

Brainard, John P.

SNL, NM/2564

(505) 844-6462

0516

jpbrain@sandia.gov

Expertise: Hydrogen ion sources, pulsed current supplies, Langmuir probes, cameras

Brockmann, John E.

SNL, NM/1512

(505) $844-3201$

0834

jebrock@sandia.gov

Expertise: Particle diagnostics

Buchenauer, D.

SNL, CA/8347

(510) $294-3570$

9162

dabuche@ca.sandia.gov

Expertise: Plasma materials interactions, plasma diagnostics, Langmuir probes

Buss, Richard

SNL, NM/1812

(505) 844-3504

0367

rjbuss@sandia.gov

Expertise: Plasma deposition and powder production, optical emission spectroscopy, time-offlight mass spectroscopy, IRIS, ECM 


\section{Personnel continued}

\begin{tabular}{|c|c|c|c|c|}
\hline Name & Facility/Dept. & Phone & $\begin{array}{l}\text { Mail } \\
\text { Stop }\end{array}$ & E-Mail Address \\
\hline Campbell, R. B. & SNL, NM/1512 & (505) $844-6205$ & 0834 & rbcampb@sandia.gov \\
\hline Expertise: & \multicolumn{4}{|c|}{$\begin{array}{l}\text { Models neutral gas flow, particle transport, fully ionized, magnetized plasma, } \\
\text { microwave propagation, plasma modeling, plasma display discharges, fusion } \\
\text { plasmas }\end{array}$} \\
\hline Causey, R. A. & SNL, CA/8347 & (510) 294-3326 & 9162 & causey@ca.sandia.gov \\
\hline
\end{tabular}

Expertise: Plasma-surface interactions, tritium plasma experiment

Chang, Chong $\mathrm{H}$.

INEL

(208) $526-2886$

3808

chc@inel.gov

Expertise: Modeling, thermal plasmas, multicomponent, multichemistry, plasma spraying, chemical processing

Choi, Seung J.

SNL, NM/1512

(505) 844-9469

0834

sjchoi@engsci.sandia.gov

Expertise: Plasma chemistry, modeling, gas phase particulate contamination

Cobble, James A.

LANL/P-4

(505) $667-8290$

E554

cobble@lanl.gov

Expertise: Spectroscopy, $x$-ray, VUV, laser scattering, fast transient visable and $x$-ray measurements via streak camera, $x$-ray image

Cook, Renita

LANL/ESA-WMA

(505) 665-0449

C930

renita@lanl.gov

Expertise: Surface treatment of abrasive powders, plasma cleaning

Cowgill, D.

SNL, CA/8347

(510) 294-2146

9162

Expertise: RF heated plasma

Dorsey, George F.

$Y-12$

(615) $574-1721$

Expertise: Plasma cleaning as a surface treatment for improved adhesion, plasma polymerization for protective coatings

Doyle, Barney L.

SNL, NM/1111

(505) 844-7568

1056

bldoyle@sandia.gov

Expertise: Plasma-surface interactions, ion sources accelerators

Dybwad, Gay

SNL, NM/2411

(505) $844-8236$

0957

gldybwa@sandia.gov

Expertise: Microwave and downstream plasma etchers, optical emission spectroscopy, circuit cleaning, high rate removal of hydrocarbons, etching of tantalum

Dykhuizen, R. C.

SNL, NM/1513

(505) 844-9105

0835

rcdykhu@sandia.gov

Expertise: Analytical modeling 


\section{Personnel continued}

\begin{tabular}{|c|c|c|c|c|}
\hline Name & Facility/Dept. & Phone & $\begin{array}{l}\text { Mail } \\
\text { Stop }\end{array}$ & E-Mail Address \\
\hline Economou, Demetre & SNL, NM/1514 & (505) 844-0124 & 0826 & economou@cfd.sandia.gov \\
\hline Expertise: & \multicolumn{4}{|c|}{$\begin{array}{l}\text { Plasma modeling and diagnostics, multidimensional simulations of plasma } \\
\text { reactors, mass spectroscopy, optical emission }\end{array}$} \\
\hline \multirow[t]{2}{*}{ Filuk, Alex B. } & SNL, NM/1271 & $(505) 845-7385$ & 1187 & abfiluk@sandia.gov \\
\hline & \multicolumn{4}{|c|}{$\begin{array}{l}\text { Pulsed-power anode/cathode plasmas, applied-B ion diodes, spectroscopic } \\
\text { diagnosis, spectral line fitting }\end{array}$} \\
\hline Funsten, Herb & LANL/NIS-1 & (505) 665-4314 & D466 & hfunsten@lanl.gov \\
\hline
\end{tabular}

Expertise: Space plasma instrumentation calibration with ion accelerator and experimental chambers for space flight sensor tests

$\begin{array}{lllll}\text { Garrett, Stephen E. } & \text { SNL, NM/2411 } & \text { (505) 845-9112 } & 0957 & \text { segarre@sandia.gov }\end{array}$

Expertise: Prebond cleaning in microelectronics packaging operations, i.e., cleaning of hybrid microcircuits

Gelbard, Fred SNL, NM/1514 (505) 844-5794 $\quad$ S $\quad$ S26 $\quad$ fgelbar@cfd.sandia.gov

Expertise: Manager, Equipment Design Support Center, providing total design capabilities for integrated circuit

Haill, Thomas A. SNL, NM/1271 (505) 845-7242 $1187 \quad$ tahaill@sandia.gov

Expertise: Modeling, ion beam transport, ion beam diagnostics

Hareland, Willard A. SNL, NM/1824 (505) 844-7758 0342

Expertise: Langmuir probe, monochromatic imaging, laser light scattering vacuum, ultraviolet spectroscopy

Harkness, John B.L. $\quad$ ANL/ES $\quad$ (708) 252-7636 363-C321 john_harkness@qmgate.anl.gov

Expertise: Microwave, plasma-chemistry, hydrogen recovery from hydrogen sulfide, waste treatment

Hassanein, Ahmed

ANL

(708) $252-5889$

207-EA133A

hassanein@anlcmt.cmt.anl.gov

Expertise: Plasma-material interactions, surface modification effects, design/develop computer codes for plasma-material interactions

Hebner, Greg

$$
\text { SNL, NM/1128 }
$$

(505) 844-6831

0607

gahebne@sandia.gov

Expertise: Gaseous electronics, etching, lighting and rare gas plasmas, laser physics, inductive and capacitive RF plasma 


\section{Personnel continued}

\begin{tabular}{|c|c|c|c|c|}
\hline Name & Facility/Dept. & Phone & $\begin{array}{l}\text { Mail } \\
\text { Stop }\end{array}$ & E-Mail Address \\
\hline Hermina, Wahid & SNL, NM/1514 & (505) $844-4759$ & 0826 & whermi@sandia.gov \\
\hline
\end{tabular}

Expertise: Manager, Equipment Design Support Center, providing total design capabilities for integrated circuit

Hunter, John A.

SNL, NM/6531

(505) 845-7865

1129

jahunte@sandia.gov

Expertise: Plasma discharges

Johannes, Justine

SNL, NM/1514

(505) 844-1994

0826

jejohan@cfd.sandia.gov

Expertise: Numerical simulation of plasma etch and deposition chemistry process using particle simulation code

Kanouff, Michael P. SNL, CA/9043 (510) 294-2105 $\quad 8743 \quad$ kanouff@california

Expertise: Particle impact and surface modeling

Koby, Martin W.

LANUCST-9

(505) 667-6011

E518

Expertise: Inductively coupled plasma mass spectrometry for multielemental analysis, environmental restoration and waste management

Krauss, Alan

$$
\text { ANL } \quad \text { (708) } 252-3520 \quad 200-D 169
$$

alan_krauss@qmgate.anl.gov

Expertise: Ion beam and plasma interactions, analytical applications of ion beams, i.e., scattering, direct recoil, mass spectroscopy

Lemke, Raymond W.

SNL, NM/1241

(505) $845-7423$

1186

rwlemke@sandia.gov

Expertise: Stability of neutral and nonneutral plasmas, electron beams in gas and vacuum, ion diodes for inertial fusion

Lopez, Edwin

SNL, NM/1815

(505) $845-9181$

0368

Expertise: Branson IPC reactor, surface preparation for enhanced adhesion and final surface cleaning

Lujan, Richard D.

$\mathrm{SNL}, \mathrm{NM} / 1332$

(505) $845-8476$

1083

lujanrd@smtplink.mdl.sandia.gov

Expertise: Chemical vapor deposition of tungsten films, in-situ NF3 plasma cleaning

Lutz, Thomas J.

SNL, NM/6531

(011)

1129

lutz@fedv09.cad.cea.fr

Expertise: Plasma materials interactions 


\section{Personnel continued}

\begin{tabular}{|c|c|c|c|c|}
\hline Name & Facility/Dept. & Phone & $\begin{array}{l}\text { Mail } \\
\text { Stop }\end{array}$ & E-Mail Address \\
\hline Martinez, Robert & SNL, NM/5932 & $844-5155$ & 0877 & \\
\hline Expertise: & \multicolumn{4}{|c|}{ Low temperature ashing, compatability, bulk material removal with high selectivity } \\
\hline McClellan, George B. & SNL, NM/1321 & (505) $844-9364$ & 0603 & \\
\hline Expertise: & \multicolumn{4}{|c|}{$\begin{array}{l}\text { Design and modification of plasma equipment, consulting on new designs, } \\
\text { modifications, upgrades }\end{array}$} \\
\hline McGrath, R. T. & SNL, NM/6503 & $(505) 844-1522$ & 0740 & rtmcgrath@pmtf.sandia.gov \\
\hline
\end{tabular}

Expertise: Plasma modeling, plasma flat panel display discharges, discharge measurements, spectroscopy, plasma etching, fusion

Meeks, Ellen

$$
\text { SNL, CA/8745 (510) 294-1459 }
$$

9042

emeeks@ca.sandia.gov

Expertise: Plasma modeling, plasma kinetics, plasma-surface interactions, software development for plasma kinetics

Mehlhorn, Thomas A.

SNL, NM/1207

(505) 845-7266

1187

temehlh@sandia.gov

Expertise: Enhanced ion stopping power, inner-shell $x$-ray generation, radiation hydrodynamics, intense ion beams

Mendel, C. W. Jr.

SNL, NM/1273

(505) $845-7492$

1194

cwmende@sandia.gov

Expertise: Microwave diagnostics, electrical diagnostics, megampere, megavolt measurements, pulsed plasma sources

Menge, Peter R.

SNL, NM/1231

(505) $845-7418$

1193

prmenge@sandia.gov

Expertise: Plasma cleaning of ion sources, charged particle beams, charged particle beam ionization of gasses

Miller, Paul A.

SNL, NM/1128

(505) $844-8879$

0601

pamille@sandia.gov

Expertise: Gaseous electronics, plasma physics, charged-particle beams, nonlinear dynamics, RF power systems

Mix, L. Paul

SNL, NM/1271

(505) 845-7493

1187

Ipmix@sandia.gov

Expertise: Numerical simulation, ICF target diagnostics, Monte Carlo simulation, $x$-ray diagnostics, holographic interferometry

Munson, Carter

LANL/P-24

(505) $665-3552$

E526

cmunson@lanl.gov

Expertise: Optical, magnetic and particle diagnostics, plasma etching, deposition and implantation, PSII users facility 


\section{Personnel continued}

\begin{tabular}{|c|c|c|c|c|}
\hline Name & Facility/Dept. & Phone & $\begin{array}{l}\text { Mail } \\
\text { Stop }\end{array}$ & E-Mail Address \\
\hline Nash, Tom & SNL, NM/1273 & (505) $845-7814$ & 0367 & tjnash@sandia.gov \\
\hline
\end{tabular}

Expertise: Plasma spectroscopy, plasma imaging, space and nsec time-resolved plasma spectroscopy, $x$-ray, etc.

Neiser, Richard A.

1841

(505) $845-3016$

1129

neiser@pmtf.sandia.gov

Expertise: Plasma spray coatings, diagnostics, modeling

Nelson, Patrick W

SNL, NM/1084

(505) 845-9777

pwneison@smtplink.mdl.sandia.gov

Expertise: RIE and plasma etching of silicon nitrides, titanium nitrides, aluminum alloys, etc., class-1 cleanrooms

Nissen, Mark

SNL, NM/2732

(505) $845-8578$

0615

Expertise: Ultra-high-speed photography and spectroscopy and other diagnostics of plasma phenomenology

$\mathrm{Ng}$, Henry

ANL

(708) 252-3992

363-b245

henry_ng@qmgate.anl.gov

Expertise: Pulsed spark to generate plasma for engines/vehicles to reduce NOx using monatomic nitrogen

Nygren, Richard E.

SNL, NM/6531

(505) $845-3135$

1129

nygren@sandia.gov

Expertise: Plasma materials interactions

O'Hern, T. J.

SNL, NM/1512

(505) $844-9061$

0834

thohern@sandia.gov

Expertise: Particle diagnostics

Oberkampf, W. L.

SNL, NM/1511

(505) $844-3799$

0825

wloberk@sandia.gov

Expertise: Computational fluid dynamics

Olson, Joseph C.

LANL/P-24

(505) 665-3193

E526

jolson@lanl.gov

Expertise: Production, characterization, manipulation, application of intense ion beams, ion beam materials processing

Pickrell, Mark M.

LANL/NIS-5

(505) 665-5098

E540

pickrell@safeguard.lanl.gov

Expertise: Alcator Tokamak and $Z \mathrm{~T}-40 \mathrm{M}$ reversed field pinch, inertial electrostatic confined plasma, nuclear "hot cell" 


\section{Personnel continued}

\begin{tabular}{|c|c|c|c|c|}
\hline Name & Facility/Dept. & Phone & $\begin{array}{l}\text { Mail } \\
\text { Stop }\end{array}$ & E-Mail Address \\
\hline Pointon, Timothy D. & SNL, NM/1242 & (505) 845-7641 & 1193 & tdpoint@sandia.go \\
\hline
\end{tabular}

Expertise: Particle-in-cell computer modeling of plasmas, large-scale 2- and 3-D electromagnetic PIC simulations

$\begin{array}{llll}\text { Poukey, James W. } & \text { SNL,NM/1241 } & \text { (505) 845-7302 } & \text { jwpouke@sandia.gov }\end{array}$

Expertise: Numerical plasma and beam modeling, transmission lines and high-current diodes, particle in-cell codes
Quintenz, Jeffrey $P$.
SNL, NM/1202
(505) $845-7245$
1195
jpquint@sandia.gov

Expertise: Numerical simulation of plasmas, particle beams and electromagnetics, highpowered electron and ion beam

Ramshaw, John

INEL

(208) $526-9240$

3808

jzr@inel.gov

Expertise: Multidimensional modeling of multicomponent thermal plasmas in LTE and nonLTE, LAVA computer code
Rej, Don
LANL/P-DO
(505) 665-1883
D434
drej@lanl.gov

Expertise: Plasma processing research facility develops plasma science and base technologies, synthesis of adyanced materials, ECM

Rieger, Dennis J. $\quad$ SNL, NM/1322 (505) 844-5554 $0603 \quad$ djriege@sandia.gov

Expertise: Plasma PR stripping, plasma etch and deposition. Hardware installation and evaluation

Riley, Merle E.

SNL, NM/1128

(505) $844-3141$

0601

meriley@sandia.gov

Expertise: Low-temperature plasma simulations and kinetics, atomic physics, molecular dynamics, scattering

Robinson, David N.

$$
\text { Y-12 (615) } 574-2983
$$

Expertise: Plasma cleaning as a surface treatment for improved adhesion, plasma polymerization for protective coatings

Rockett, Paul D.

SNL, NM/6531

(505) $845-7466$

1129

pdrocke@sandia.gov

Expertise: High-density plasma, magnetic fusion disruption simulation, $x$-ray, EUV, UV spectroscopy

Rosocha, Louis

LANL/CST-18

(505) 667-8493

E525

rosocha@cst4.lanl.gov

Expertise: Atmospheric pressure nonthermal plasmas, environmental applications, plasma chemistry modeling, chemical processing 


\section{Personnel continued}

\begin{tabular}{|c|c|c|c|c|}
\hline Name & Facility/Dept. & Phone & $\begin{array}{l}\text { Mail } \\
\text { Stop }\end{array}$ & E-Mail Address \\
\hline Rowton, Larry J. & LANL/P-15 & (505) 667-5211 & $\mathrm{K} 480$ & ljrowton@lanl.gov \\
\hline
\end{tabular}

Russo, Anthony J.

SNL, NM/6531

(301) $903-6288$

1129

anthony.russo@mailgw.er.doe.gov

Expertise: Runaway electron generation

Sandoval, Cindy

LANL/ESA-WMA

(505) $667-8393$

C930

cws@lanl.gov

Expertise: Cleaning, plasma deposition, surface modification

Sanford, T. W. L.

SNL, NM/1231

(505) 845-7816

Expertise: Electron diodes, intense electron beam transport, Hermes III calorimeters, fast framing $x$-ray camera

Selwyn, Gary S.

LANL/P-24

(505) 665-7359

E526

gss@fjwsys.lanl.gov

Expertise: Real-time in-situ detection and control of particulate contamination, design modification for process uniformity

Shagan, Richard

SNL, NM/2732

(505) 845-9079

0615

Expertise: Ultra-high-speed photography and spectroscopy and other diagnostics of plasma phenomenolgy

Shul, Randy J.

SNL, NM/1322

(505) 844-6126

0603

rishul@somnet.sandia.gov

Expertise: Barrel asher, RIE, PECVD, ECR, refractory metals, dielectrics, phosphide, arsenic, and nitride-based III-V compound semiconductors

Smith, David Lewis

SNL, NM/1239

(505) $845-7141$

1184

Expertise: High-voltage, high-current gas switching, high-current electron beam diode development, laser triggered

Smith, Mark F.

SNL, NM/1841

(505) $845-3256$

1129

mfsmith@pmtf.sandia.gov

Expertise: Plasma spray coatings, diagnostics, modeling

Smith, Mike

SNL, NM/1812

(505) 845-7762

0367

Expertise: Plasma cleaning, etching, deposition, powder production, lasers, optical emission spectroscopy, FTIR, Dektak, etc. 


\section{Personnel continued}

\begin{tabular}{|c|c|c|c|c|}
\hline Name & Facility/Dept. & Phone & $\begin{array}{l}\text { Mail } \\
\text { Stop }\end{array}$ & E-Mail Address \\
\hline Stevens, J. E. & & (505) 844-2802 & 1077 & $\begin{array}{c}\text { James_E._Stevens@smtplink. } \\
\text { mdl.sandia.gov }\end{array}$ \\
\hline Expertise: & \multicolumn{4}{|c|}{$\begin{array}{l}\text { Plasma and RF diagnostics, RFI/ICP, ECR, helicon, diode and microwave plasma } \\
\text { reactor }\end{array}$} \\
\hline Tsai, c. c. (Jim) & $\frac{\gamma-12}{\gamma-1}$ & (615) $574-1124$ & & \\
\hline
\end{tabular}

Expertise: RF, AC- or DC-biased microwave plasma and plasma shaping with magnets

Tuszewski, Michel

LANL/P24

(505) 667-3566

E526

mgtu@lanl.gov

Expertise: Plasma experimentalist, plasma diagnostics, plasma source development, and modeling

Ulrickson, Michael A. SNL, NM/6531 (505) 845-3020 $1129 \quad$ maulric@sandia.gov

Expertise: Edge plasma physics, plasma impurity control, plasma materials interactions

Veerasingam, $\mathbf{R}$

SNL, NM/6531

(505) $845-3490$

ramana@pmtf.sandia.gov

Expertise: Plasma modeling, plasma display discharges, fusion plasmas

Walsh, David S. $\quad$ SNL, NM/1111 (505) 844-9590 $1056 \quad$ dswalsh@sandia.gov

Expertise: Plasma surface interactions, ion sources accelerators

$\begin{array}{lllll}\text { Wampler, William R. } & \text { SNL, NM/1111 } & \text { (505) 844-4114 } & 1056 \quad \text { wrwampl@sandia.gov }\end{array}$

Expertise: Plasma surface interactions, ion sources accelerators
Ward, Pamela P.
$\because \%$
$\mathrm{SNL}, \mathrm{NM} / 1812$
(505) $844-2038$
0367
ppward@sandia.gov

Expertise: Plasma cleaning, etching, RF inductive and capacitive apparatus, optical emission spectroscopy, interferometry, patterning

Watkins, Jonathan G. SNL, NM/6531 (619) 455-3670 $1129 \quad$ watkins@gav.gat.com

Expertise: Langmuir probes, edge plasma physics
Weatherspoon, Kim A.
$\gamma-12$
(615) $576-4228$

Expertise: Plasma cleaning as a surface treatment for improved adhesion, plasma polymerization for protective coatings
Wilson, Ken
SNL, CA/8304
(510) $294-2497$
9163
klwilso@ca.sandia.gov

Expertise: Magnetic fusion energy, plasma-surface interactions 


\section{Personnel concluded}

\begin{tabular}{|c|c|c|c|c|}
\hline Name & Facility/Dept. & Phone & $\begin{array}{l}\text { Mail } \\
\text { Stop }\end{array}$ & E-Mail Address \\
\hline Wood, Blake P. & LANL/P-24 & (505) 665-6524 & E536 & bwood@lanl.gov \\
\hline Expertise: & \multicolumn{4}{|c|}{$\begin{array}{l}\text { Low-pressure, low-temperature plasma discharge for etching or deposition, } \\
\text { cathode arc technology, particle-in-cell models }\end{array}$} \\
\hline Youchison, Dennis L. & SNL, NM/6531 & (505) $845-3138$ & $\begin{array}{c}\therefore \\
1129\end{array}$ & dlyouch@sandia.gov \\
\hline
\end{tabular}




\section{Plasma Technology Directory}

\section{Laboratories}

\section{Argonne National Laboratory (ANL)}

\begin{tabular}{|c|c|c|c|c|}
\hline Name & Dept. & Phone & $\begin{array}{l}\text { Mail } \\
\text { Stop }\end{array}$ & E-Mail Address \\
\hline
\end{tabular}

Harkness, John B.L. ES (708) 252-7636 363-C321 john_harkness@gmgate.anl.gov

Expertise: Microwave, plasma-chemistry, hydrogen recovery from hydrogen sulfide, waste treatment

Hassanein, Ahmed

(708) 252-5889 207-EA133A hassanein@anlcmt.cmt.anl.gov

Expertise: Plasma-material interactions, surface modification effects, design/develop computer codes for plasma-material interactions

Krauss, Alan

(708) 252-3520 207-EA133A alan_krauss@qmgate.ani.gov

Expertise: Ion beam and plasma-surface interactions, analytical applications of ion beams, i.e., scattering, direct recoil, mass spectroscopy

$\mathrm{Ng}$, Henry

(708) 252-3992 363-b245

henry_ng@qmgate.anl.gov

Expertise: Pulsed spark to generate plasma for engines/vehicles to reduce N0x using monatomic nitrogen

\section{Idaho National Engineering Laboratory (INEL)}

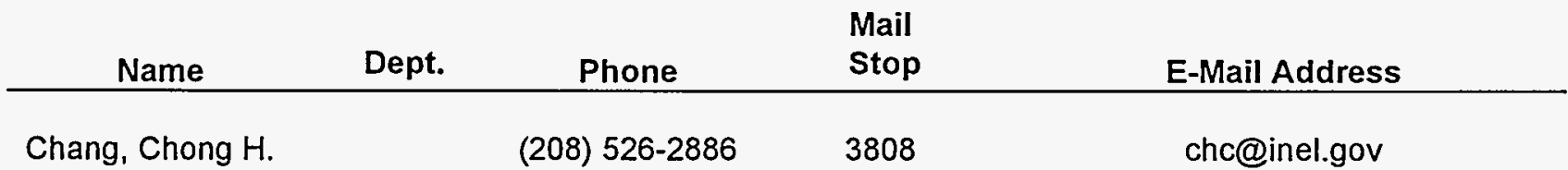

Expertise: Modeling, thermal plasmas, multicomponent, multichemistry, plasma spraying, chemical processing

Ramshaw, John

(208) $526-9204$

3808

jzr@inel.gov

Expertise: Multidimensional modeling of multicomponent thermal plasmas in LTE and nonLTE, LAVA computer code 


\section{Los Alamos National Laboratory (LANL)}

\begin{tabular}{|c|c|c|c|c|}
\hline Name & Dept. & Phone & $\begin{array}{l}\text { Mail } \\
\text { Stop }\end{array}$ & E-Mail Address \\
\hline Cobble, James A. & $P-4$ & (505) 667-8290 & E554 & cobble@lanl.gov \\
\hline Expertise: & \multicolumn{4}{|c|}{$\begin{array}{l}\text { Spectroscopy, } x \text {-ray, VUV, laser scattering, fast transient visable and } x \text {-ray } \\
\text { measurements via streak camera, } x \text {-ray image }\end{array}$} \\
\hline Cook, Renita & ESA-WMA & (505) 665-0449 & $\mathrm{C} 930$ & renita@lanl.gov \\
\hline Expertise: & \multicolumn{4}{|c|}{ Surface treatment of abrasive powders, plasma cleaning } \\
\hline Funsten, Herb & NIS-1 & (505) 665-4314 & D466 & hfunsten@lanl.gov \\
\hline
\end{tabular}

Expertise: Space plasma instrumentation calibration with ion accelerator and experimental chambers for space flight sensor tests

Koby, Martin W. $\quad$ CST-9 (505) 667-6011 E518

Expertise: Inductively coupled plasma mass spectrometry for multielemental analysis, environmental restoration and waste management

Munson, Carter

$\mathrm{P}-24$

(505) 665-3552

E526

cmunson@lanl.gov

Expertise: Optical, magnetic and particle diagnostics, plasma etching, deposition and implantation, PSII users facility

Olson, Joseph C.

$P-24$

(505) 665-3193

E526

jolson@lanl.gov

Expertise: Production, characterization, manipulation, application of intense ion beams, ion beam materials processing

Pickrell, Mark M. NIS-5 (505) 665-5098 E540 $\quad$ pickrell@safeguard.lanl.gov

Expertise: Alcator Tokamak and ZT-40M reversed field pinch, inertial electrostatic confined plasma, nuclear "hot cell"

Rej, Don

P-DO

(505) $665-1883$

D434

drej@lanl.gov

Expertise: Plasma processing research facility develops plasma science and base technologies, synthesis of advanced materials, ECM

Rosocha, Louis

CST-18 (505) 667-8493

E525

rosocha@cst4.Ianl.gov

Expertise: Atmospheric pressure nonthermal plasmas, environmental applications, plasma chemistry modeling, chemical processing 
LANL, continued

\begin{tabular}{|c|c|c|c|c|}
\hline Name & Dept. & Phone & $\begin{array}{l}\text { Mail } \\
\text { Stop }\end{array}$ & E-Mail Address \\
\hline Rowton, Larry J. & $P-15$ & (505) 667-5211 & $\mathrm{K} 480$ & ljrowton@lanl.gov \\
\hline Expertise: & \multicolumn{4}{|c|}{$\begin{array}{l}\text { Ion beam facility, ion accelerators with highly collimated and energy-resolved } \\
\text { beams with energies from } 20 \mathrm{keV} \text { to } 150 \mathrm{MeV}\end{array}$} \\
\hline Sandoval, Cindy & ESA-WMA & (505) 667-8393 & $\mathrm{C} 930$ & cws@lanl.gov \\
\hline Expertise: & \multicolumn{4}{|c|}{ Cleaning, plasma deposition, surface modification } \\
\hline Selwyn, Gary S. & $P-24$ & $(505) 665-7359$ & $E 526$ & gss@fjwsys.lanl.gov \\
\hline Expertise: & \multicolumn{4}{|c|}{$\begin{array}{l}\text { Real-time in-situ detection and control of particulate contamination, design } \\
\text { modification for process uniformity }\end{array}$} \\
\hline Tuszewski, Michel & $P-24$ & (505) 667-3566 & E526 & mgtu@lanl.gov \\
\hline Expertise: & \multicolumn{4}{|c|}{$\begin{array}{l}\text { Plasma experimentalist, plasma diagnostics, plasma source development, and } \\
\text { modeling }\end{array}$} \\
\hline Wood, Blake P. & $P-24$ & (505) 665-6524 & E536 & bwood@lanl.gov \\
\hline Expertise: & \multicolumn{4}{|c|}{$\begin{array}{l}\text { Low-pressure, low-temperature plasma discharge for etching or deposition, } \\
\text { cathode }\end{array}$} \\
\hline
\end{tabular}

\section{Sandia National Laboratories, California (SNL, CA)}

\begin{tabular}{|c|c|c|c|c|}
\hline Name & Dept. & Phone & $\begin{array}{l}\text { Mail } \\
\text { Stop }\end{array}$ & E-Mail Address \\
\hline Bastasz, R. & 8347 & (510) 294-2013 & 9162 & bastasz@vaxm.gat.com \\
\hline $\begin{array}{c}\text { Expertise: } \\
\because \because\end{array}$ & \multicolumn{4}{|c|}{ Plasma-surface interactions, SIMS, AES, ISS } \\
\hline Buchenauer, D. A. & 8347 & (510) 294-3570 & 9162 & dabuche@ca.sandia.gov \\
\hline $\begin{array}{l}\text { Expertise: } \\
\cdots\end{array}$ & \multirow{2}{*}{\multicolumn{4}{|c|}{$\begin{array}{l}\text { Plasma materials interactions, plasma diagnostics, Languir probes } \\
\begin{array}{lccc}8347 & \text { (510) 294-3326 } & 9162 & \text { causey@ca.sandia.gov }\end{array}\end{array}$}} \\
\hline Causey, R. A. & & & & \\
\hline Expertise: & \multicolumn{4}{|c|}{ Plasma-surface interactions, tritium plasma experiment } \\
\hline
\end{tabular}

Expertise: RF heated plasma 
SNL, CA concluded

\begin{tabular}{ccccc} 
Name & Dept. & Phone & $\begin{array}{c}\text { Mail } \\
\text { Stop }\end{array}$ & E-Mail Address \\
\hline Kanouff, Michael P. & 9043 & $(510) 294-2105$ & 8743 & kanouff@california \\
Expertise: & Particle impact and surface modeling & \\
Meeks, Ellen & 8745 & $(510) 294-1459$ & 9042 & emeeks@ca.sandia.gov \\
Expertise: & $\begin{array}{l}\text { Plasma modeling, plasma kinetics, plasma-surface interactions, software } \\
\text { development for plasma kinetics }\end{array}$ & \\
Wilson, Ken & 8304 & (510) 294-2497 & 9163 & klwilso@ca.sandia.gov
\end{tabular}

Expertise: Magnetic fusion energy, plasma-surface interactions

\section{Sandia National Laboratories, New Mexico (SNL, NM)}

\begin{tabular}{rcccc} 
Name & Dept. & Phone & $\begin{array}{c}\text { Mail } \\
\text { Stop }\end{array}$ & E-Mail Address \\
\hline Apblett, Chris & 1323 & $(505) 844-4487$ & & apbletca@sandia.gov
\end{tabular}

Expertise: Electron cyclotron resonance system capable of depositing thin films on 6-inch silicon substrates

Bailey, James E. $\quad 1277 \quad(505)$ 845-7203 $1196 \quad$ jebaile@sandia.gov

Expertise: Z-pinch, laser-produced, and ion diode plasmas, plasma spectroscopy, time and space resolved spectra

$\begin{array}{lllll}\text { Barbour, J. Charles } & 1111 & (505) \text { 844-5517 jcbarbo@sandia.gov }\end{array}$

Expertise: Plasma assisted deposition, ECR accelerations

$\begin{array}{lllll}\text { Bartel, Tim } & 1514 & (505) 844-0124 & 0826 \quad \text { tjbarte@cfd.sandia.gov }\end{array}$

Expertise: Numerical simulation of plasma etch and deposition reactors and processes by self-consistent particle method

$\begin{array}{lllll}\text { Blackwell, Bennie } & 1514 & \text { (505) 845-8844 blblack@sandia.gov }\end{array}$

Expertise: Modeling of thermal conduction, convection and radiation processes to design and improve plasma equipment

$\begin{array}{lllll}\text { Brainard, John P. } & 2564 & \text { (505) 844-6462 } & 0516 \quad \text { jpbrain@sandia.gov }\end{array}$

Expertise: Hydrogen ion sources, pulsed current supplies, Langmuir probes, cameras 


\section{SNL, NM continued}

\begin{tabular}{|c|c|c|c|c|}
\hline Name & Dept. & Phone & $\begin{array}{l}\text { Mail } \\
\text { Stop }\end{array}$ & E-Mail Address \\
\hline Brockmann, John E. & 1512 & (505) 844-3201 & 0834 & jebrock@sandia.gov \\
\hline Expertise: & \multicolumn{4}{|c|}{ Particle diagnostics } \\
\hline …. \% \% & 1812 & था० 210 & 0367 & \%: $\quad \because \quad$ : \\
\hline
\end{tabular}

Expertise: Plasma deposition and powder production, optical emission spectroscopy, timeof-flight mass spectroscopy, IRIS, ECM
Campbell, R. B.
1512
(505) 844-6205
0834
rbcampb@sandia.gov

Expertise: Models neutral gas flow, particle transport, fully ionized magnetized plasma, microwave propagation, plasma modeling, plasma display discharges, fusion plasmas
Choi, Seung J.
1512
(505) 844-9469
0834
sjchoi@engsci.sandia.gov

Expertise: Plasma chemistry, modeling, gas phase particulate contamination

$\begin{array}{lllll}\text { Doyle, Barney L. } & 1111 & \text { (505) } 844-7568 & 1056 & \text { bldoyle@sandia.gov }\end{array}$

Expertise: Plasma surface interactions, ion sources accelerators
Dybwad, Gay
2411
(505) $844-8236$
0957
gldybwa@sandia.gov

Expertise: Microwave and downstream plasma etchers, optical emission spectroscopy, circuit cleaning, high rate removal of hydrocarbons, etching of tantalum
Dykhuizen, R. C.
1513
(505) 844-9105
0835
rcdykhu@sandia.gov

Expertise: Analytical modeling

Economou, Demetre $\quad 1514 \quad(505)$ 844-0124 $0826 \quad$ economou@cfd.sandia.gov

Expertise: Plasma modeling and diagnostics, multidimensional simulations of plasma reactors, mass spectroscopy, optical emission

Filuk, Alex B.

$$
1271 \text { (505) 845-7385 } 1187 \text { abfiluk@sandia.gov }
$$

Expertise: Pulsed-power anode/cathode plasmas, applied-B ion diodes, spectroscopic diagnosis, spectral line fitting
Garrett, Stephen E.
2411
(505) $845-9112$
0957 segarre@sandia.gov

Expertise: Prebond cleaning in microelectronics packaging operations, i.e., cleaning of hybrid microcircuits 
SNL, NM continued

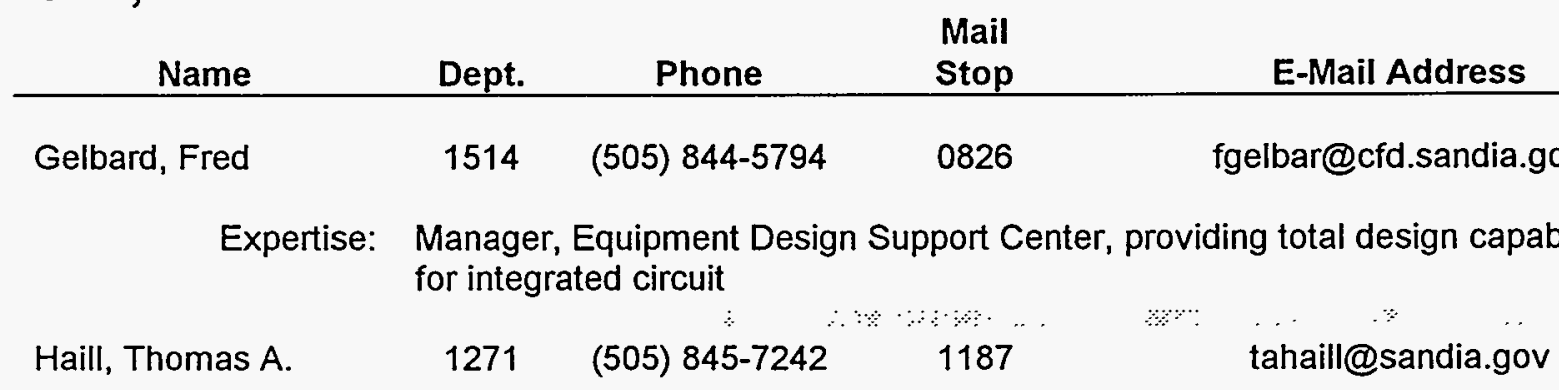

Expertise: Modeling, ion beam transport, ion beam diagnostics

Hareland, Willard A. $1824 \quad$ (505) 844-7758 0342

Expertise: Langmuir probe, monochromatic imaging, laser light scattering vacuum, ultraviolet spectroscopy

$\begin{array}{lllll}\text { Hebner, Greg } & 1128 & \text { (505) } 844-6831 & 0607 & \text { gahebne@sandia.gov }\end{array}$

Expertise: Gaseous electronics, etching, lighting and rare gas plasmas, laser physics, inductive, capacitive RF plasma

$\begin{array}{lllll}\text { Hermina, Wahid } & 1514 & (505) \text { 844-4759 } & 0826 & \text { whermi@sandia.gov }\end{array}$

Expertise: Manager, Equipment Design Support Center, providing total design capabilities for integrated circuit

Hunter, John A.

$6531 \quad(505) 845-7865$

1129

jahunte@sandia.gov

Expertise: Plasma discharges

Johannes, Justine $\quad 1514 \quad$ (505) 844-1994 $0826 \quad$ jejohan@cfd.sandia.gov

Expertise: Numerical simulation of plasma etch and deposition chemistry processes using particle simulation code

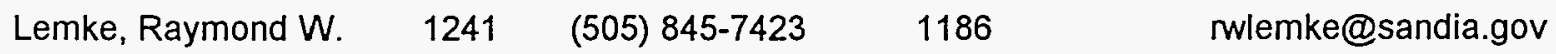

Expertise: Stability of neutral and nonneutral plasmas, electron beams in gas and vacuum, ion diodes for inertial fusion

Lopez, Edwin $\quad 1815 \quad(505) 845-9181 \quad 0368$

Expertise: Branson IPC reactor, surface preparation for enhanced adhesion and final surface cleaning

Lujan, Richard D. $\quad 1332 \quad$ (505) 845-8476 $1083 \quad$ lujanrd@smtplink.mdl.sandia.gov

Expertise: Chemical vapor deposition of tunsten films, in-situ NF3 plasma cleaning 
SNL, NMI continued

\begin{tabular}{ccccc} 
Name & Dept. & Phone & $\begin{array}{c}\text { Mail } \\
\text { Stop }\end{array}$ & E-Mail Address \\
\hline Lutz, Thomas J. & 6531 & $\begin{array}{c}(011) \\
33-9079-5105\end{array}$ & 1129 & lutz@fedv09.cad.cea.fr
\end{tabular}

Expertise: Plasma materials interactions

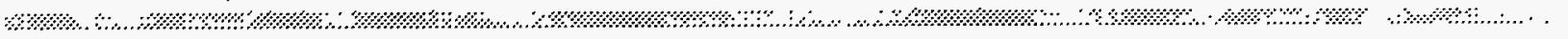

$\begin{array}{lll}\text { Martinez, Robert } \quad 5932 & \mathbf{8 4 4 - 5 1 5 5} & 0877\end{array}$

Expertise: Low temperature ashing, compatability, bulk material removal with high selectivity

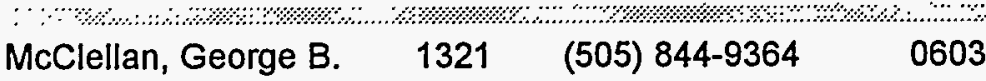

Expertise: Design and modification of plasma equipment, consulting on new designs, modifications, upgrades

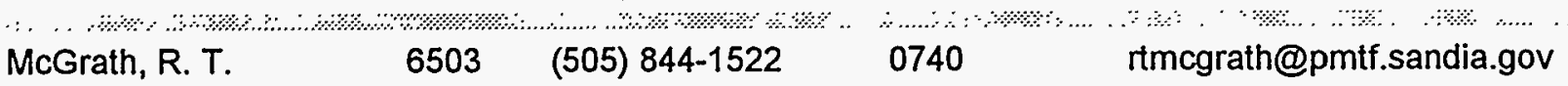

Expertise: Plasma modeling, plasma flat panel display discharges, discharge measurements, spectroscopy, plasma etching, fusion

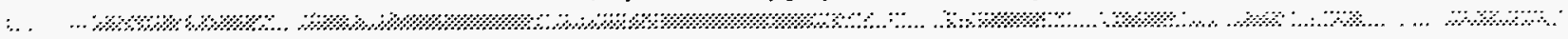

$\begin{array}{lllll}\text { Mehlhorn, Thomas A. } & 1207 & \text { (505) 845-7266 } & 1187 & \text { temehlh@sandia.gov }\end{array}$

Expertise: Enhanced ion stopping power, inner-shell x-ray generation, radiation hydrodynamics, intense ion beams

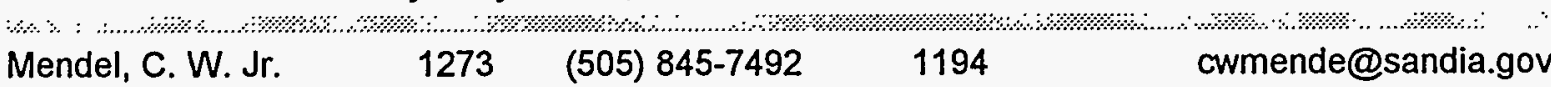

Expertise: Microwave diagnostics, electrical diagnostics, megampere, megavolt measurements, pulsed plasma sources

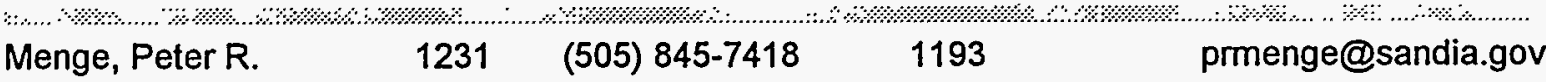

Expertise: Plasma cleaning of ion sources, charged particle beams, charged particle beam ionization of gasses

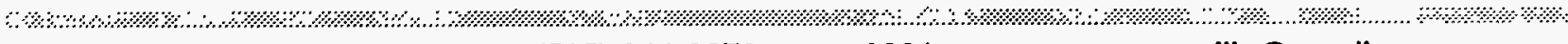

Miller, Paul A. $\quad 1128 \quad(505) 844-8879 \quad 0601 \quad$ pamille@sandia.gov

Expertise: Gaseous electronics, plasma physics, charged-particle beams, nonlinear dynamics, RF power systems
Mix, L. Paul
1271
(505) 845-7493
1187
Ipmix@sandia.gov

Expertise: Numerical simulation, ICF target diagnostics, Monte Carlo simulation, $x$-ray diagnostics, holographic interferometry

$\begin{array}{lllll}\text { Nash, Tom } & 1273 & (505) 845-7814 & 0367 & \text { tjnash@sandia.gov }\end{array}$

Expertise: Plasma spectroscopy, plasma imaging, space and nsec time-resolved plasma spectroscopy, x-ray, etc. 
SNL, NM continued

\begin{tabular}{|c|c|c|c|c|}
\hline Name & Dept. & Phone & $\begin{array}{l}\text { Mail } \\
\text { Stop }\end{array}$ & E-Mail Address \\
\hline Neiser, Richard A. & 1841 & (505) $845-3016$ & 1129 & neiser@pmtf.sandia.gov \\
\hline Expertise: & \multicolumn{4}{|c|}{ Plasma spray coatings, diagnostics, modeling } \\
\hline Nelson, Patrick W. & 1084 & (505) $845-9777$ & & pwnelson@smtplink.mdl.sandia.gov \\
\hline Expertise: & \multicolumn{4}{|c|}{$\begin{array}{l}\text { RIE and plasma etching of silicon nitrides, titanium nitrides, aluminum alloys, } \\
\text { etc., class- } 1 \text { cleanrooms }\end{array}$} \\
\hline Nissen, Mark & 2732 & $(505) 845-8578$ & 0615 & \\
\hline Expertise: & \multicolumn{4}{|c|}{$\begin{array}{l}\text { Ultra-high-speed photography and spectroscopy and other diagnostics of plasma } \\
\text { phenomenology }\end{array}$} \\
\hline Nygren, Richard E. & 6531 & (505) $845-3135$ & 1129 & nygren@sandia.gov \\
\hline $\begin{array}{l}\text { Expertise: } \\
\text { O'Hern, Timothy }\end{array}$ & $\begin{array}{c}\text { Plasma } \\
1512\end{array}$ & $\begin{array}{r}\text { aterials interactior } \\
(505) \text { 844-9061 }\end{array}$ & 0834 & thohern@sandia.gov \\
\hline
\end{tabular}

Expertise: Particle diagnostics

$\begin{array}{lllll}\text { Oberkampf, W. L. } & 1511 & \text { (505) 844-3799 } & 0825 & \text { wloberk.sandia.gov }\end{array}$

Expertise: Computational fluid dynamics

Pointon, Timothy D. $1242 \quad$ (505) 845-7641 $1193 \quad$ tdpoint@sandia.gov

Expertise: Particle-in-cell computer modeling of plasmas, large-scale 2- and 3-D electromagnetic PIC simulations

Poukey, James W. $1241 \quad$ (505) 845-7302 jwpouke@sandia.gov

Expertise: Numerical plasma and beam modeling, transmission lines and high-current diodes, particle in-cell codes

Quintenz, Jeffrey P. $1202 \quad$ (505) 845-7245 $1195 \quad$ jpquint@sandia.gov

Expertise: Numerical simulation of plasmas, particle beams and electromagnetics, highpowered electron and ion beam

Rieger, Dennis J. $\quad 1322 \quad(505)$ 844-5554 $0603 \quad$ djriege@sandia.gov

Expertise: Plasma PR stripping, plasma etch and deposition. Hardware installation and evaluation

Riley, Merle E. $\quad 1128 \quad$ (505) 844-3141 $0601 \quad$ meriley@somnet.sandia.gov

Expertise: Low-temperature plasma simulations and kinetics, atomic physics, molecular dynamics, scattering 
SNL, NM continued

\begin{tabular}{|c|c|c|c|c|}
\hline Name & Dept. & Phone & $\begin{array}{l}\text { Mail } \\
\text { Stop }\end{array}$ & E-Mail Address \\
\hline Rockett, Paul D. & 6531 & (505) 845-7466 & 1129 & pdrocke@sandia.gov \\
\hline Expertise: & \multicolumn{4}{|c|}{$\begin{array}{l}\text { High-density plasma, magnetic fusion disruption simulation, x-ray, EUV, UV } \\
\text { spectroscopy }\end{array}$} \\
\hline$\therefore \quad: \quad: \quad: \% \% \% \% \% \%$ & \%\% & $\because \%$ & $\%$ & $\% \%$ \\
\hline Russo, Anthony J. & 6531 & (301) 903-6288 & 1129 & anthony.russo@mailgw.er.doe.gov \\
\hline Expertise: & \multicolumn{4}{|c|}{ Runaway electron generation } \\
\hline Sanford, T. W. L. & 1231 & (505) $845-7816$ & & \\
\hline
\end{tabular}

Expertise: Electron diodes, intense electron beam transport, Hermes III, calorimeters, fast framing $x$-ray camera

\%. Shagan, Richard $\quad 2732 \quad(505) 845-9079 \quad 0615$

Expertise: Ultra-high-speed photography and spectroscopy and other diagnostics of plasma phenomenology

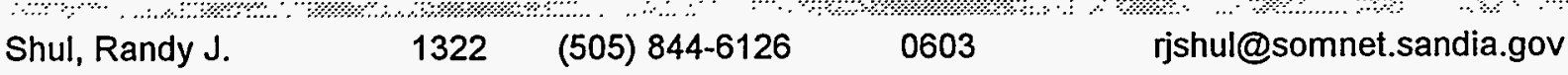

Expertise: Barrel asher, RIE, PECVD, ECR, refractory metals, dielectrics, phosphide, arsenic, and nitride-based III-V compound semiconductors

Smith, David Lewis $1239 \quad$ (505) 845-7141 1184

Expertise: High-voltage, high-current gas switching, high-current electron beam diode development, laser triggered

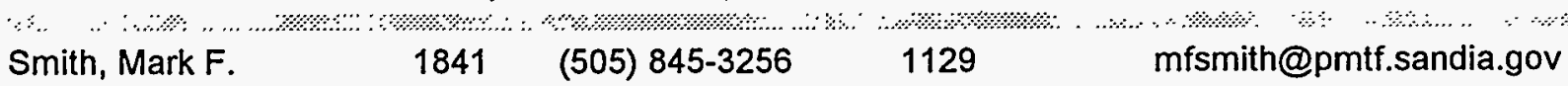

Expertise: Plasma spray coatings, diagnostics, modeling

$\begin{array}{llll}\text { Smith, Mike } & 1812 & (505) 845-7762 & 0367\end{array}$

Expertise: Plasma cleaning, etching, deposition, powder production, lasers, optical emission spectroscopy, FTIR, Dektak, etc.

Stevens, J. E. $\quad 1326 \quad(505)$ 844-2802 $1077 \quad$ James_E._Stevens@smtplink.mdl.sandia.gov

Expertise: Plasma and RF diagnostics, RFI/ICP, ECR, helicon, diode and microwave plasma reactor

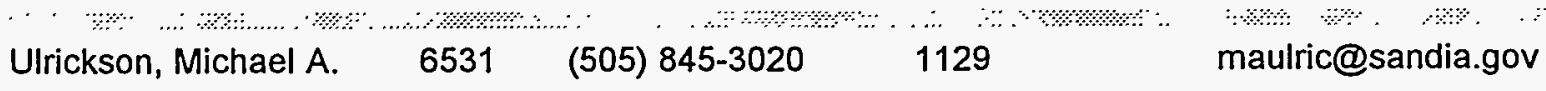

Expertise: Edge plasma physics, plasma impurity control, plasma materials interactions

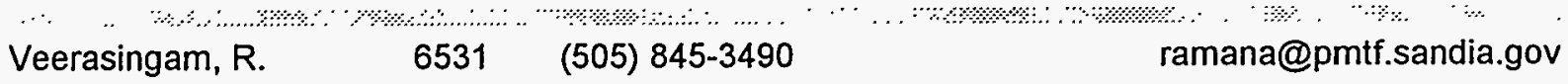

Expertise: Plasma modeling, plasma display discharges, fusion plasmas 
SNL, NM concluded

\begin{tabular}{|c|c|c|c|c|}
\hline Name & Dept. & Phone & $\begin{array}{l}\text { Mail } \\
\text { Stop }\end{array}$ & E-Mail Address \\
\hline Walsh, David S. & 1111 & (505) $844-9590$ & 1056 & dswalsh@sandia.gov \\
\hline $\begin{array}{r}\text { Expertise: } \\
\text { Wampler, William R. }\end{array}$ & \multicolumn{4}{|c|}{$\begin{array}{l}\text { Plasma surface interactions, ion sources accelerators } \\
\begin{array}{ccccc}1111 & (505) 844-4114 & 1056 & \text { wrwampl@sandia.gov }\end{array}\end{array}$} \\
\hline $\begin{array}{r}\text { Expertise: } \\
\text { Ward Pamela } P\end{array}$ & $\begin{array}{l}\text { Plasma } \\
\therefore \\
1812\end{array}$ & $\begin{array}{r}\text { rface interactions } \\
\quad\end{array}$ & source & $\begin{array}{l}\text { rs } \\
\therefore \text { nnwardesandia gov }\end{array}$ \\
\hline Expertise: & \multicolumn{4}{|c|}{$\begin{array}{l}\text { Plasma cleaning, etching, RF inductive and capacitive apparatus, optical } \\
\text { emission spectroscopy, interferometry, patterning }\end{array}$} \\
\hline Watkins, Jonathan G. & 6531 & (619) $455-3670$ & 1129 & watkins@gav.gat.com \\
\hline $\begin{array}{l}\text { Expertise: } \\
\text { Youchison, Dennis L. }\end{array}$ & $\begin{array}{l}\text { Langmi } \\
6531\end{array}$ & $\begin{array}{l}\text { orobes, edge plas } \\
\because \\
(505) 845-3138\end{array}$ & $\begin{array}{l}\text { hysics } \\
1129\end{array}$ & dlyouch@sandia.gov \\
\hline Expertise: & \multicolumn{4}{|c|}{ ECR chemical vapor deposition, ion implantation } \\
\hline$Y-12$ & & & & \\
\hline Name & & Phone & & \\
\hline
\end{tabular}

Dorsey, George F.

(615) $574-1721$

Expertise: Plasma cleaning as a surface treatment for improved adhesion, plasma polymerization for protective coatings

Robinson, David N.

(615) $574-2983$

Expertise: Plasma cleaning as a surface treatment for improved adhesion, plasma polymerization for protective coatings

Tsai, C. C. (Jim)

(615) $574-1124$

Expertise: RF, AC- or DC-biased microwave plasma and plasma shaping with magnets

Weatherspoon, Kim A.

(615) $576-4228$

Expertise: Plasma cleaning as a surface treatment for improved adhesion, plasma polymerization for protective coatings 


\section{Plasma Technology Directory}

\section{Technologies}

\section{Accelerators}

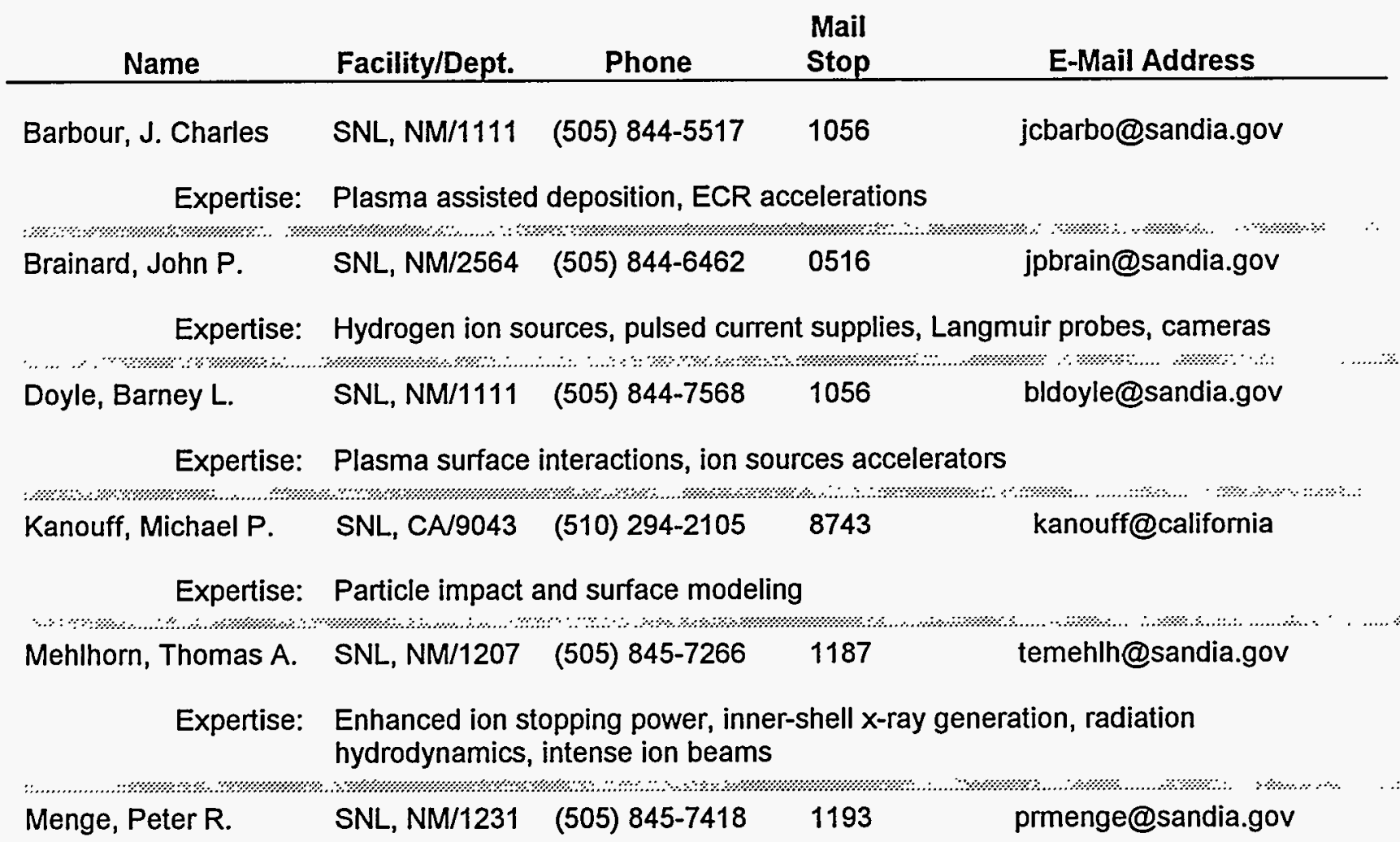

Expertise: Plasma cleaning of ion sources, charged particle beams, charged particle beam ionization of gasses

$\begin{array}{lllll}\text { Miller, Paul A. } & \text { SNL, NM/1128 } & (505) \text { 844-8879 } & 0601 & \text { pamille@sandia.gov }\end{array}$

Expertise: Gaseous electronics, plasma physics, charged-particle beams, nonlinear dynamics, RF power systems

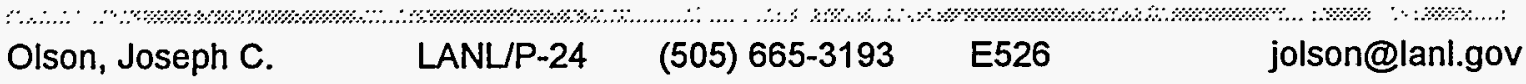

Expertise: Production, characterization, manipulation, application of intense ion beams, ion beam materials processing

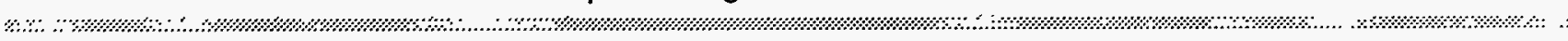

$\begin{array}{llll}\text { Pickrell, Mark M. } & \text { LANL/NIS-5 } & \text { (505) 665-5098 E540 } & \text { pickrell@safeguard.lanl.gov }\end{array}$

Expertise: Alcator Tokamak and ZT-40M reversed field pinch, inertial electrostatic confined plasma, nuclear "hot cell" 


\section{Accelerators concluded}

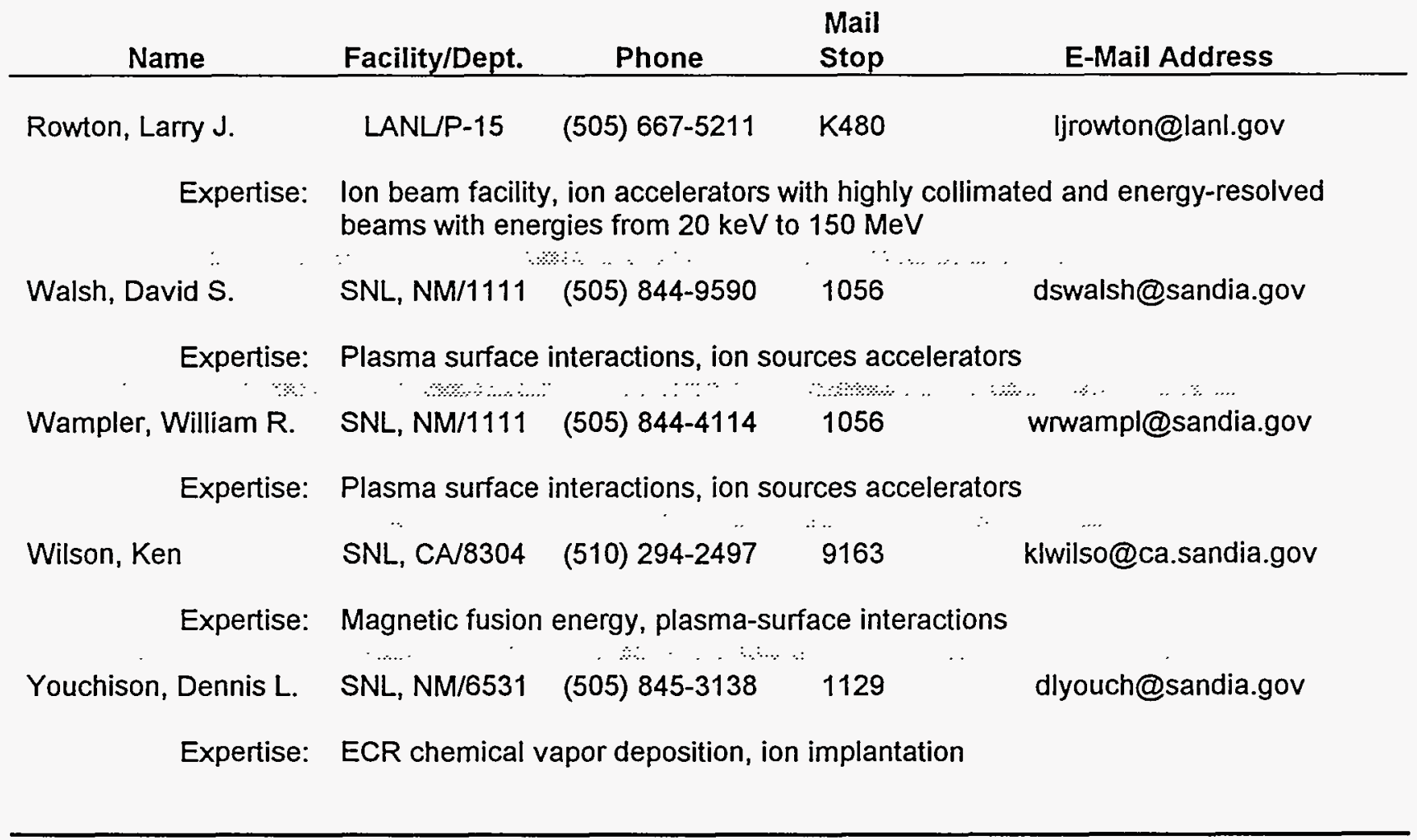

\section{Cleaning and Etching}

\begin{tabular}{|c|c|c|c|c|}
\hline Name & Facility/Dept. & Phone & $\begin{array}{l}\text { Mail } \\
\text { Stop }\end{array}$ & E-Mail Address \\
\hline Cook, Renita & LANL/ESA-WMA & 5) $665-0449$ & C930 & renita@lanl.gov \\
\hline
\end{tabular}

Expertise: Surface treatment of abrasive powders, plasma cleaning

Dorsey, George F.

$$
Y-12
$$$$
\text { (615) } 574-1721
$$

Expertise: Plasma cleaning as a surface treatment for improved adhesion, plasma polymerization for protective coatings

Dybwad, Gay

$$
\text { SNL, NM/2411 (505) 844-8236 } 0957 \quad \text { gldybwa@sandia.gov }
$$

Expertise: Microwave and downstream plasma etchers, optical emission spectroscopy, circuit cleaning, high rate removal of hydrocarbons, etching of tantalum

Garrett, Stephen E. SNL, NM/2411 (505) 845-9112 $0957 \quad$ segarre@sandia.gov

Expertise: Prebond cleaning in microelectronics packaging operations, i.e., cleaning of hybrid microcircuits 


\section{Cleaning and Etching continued}

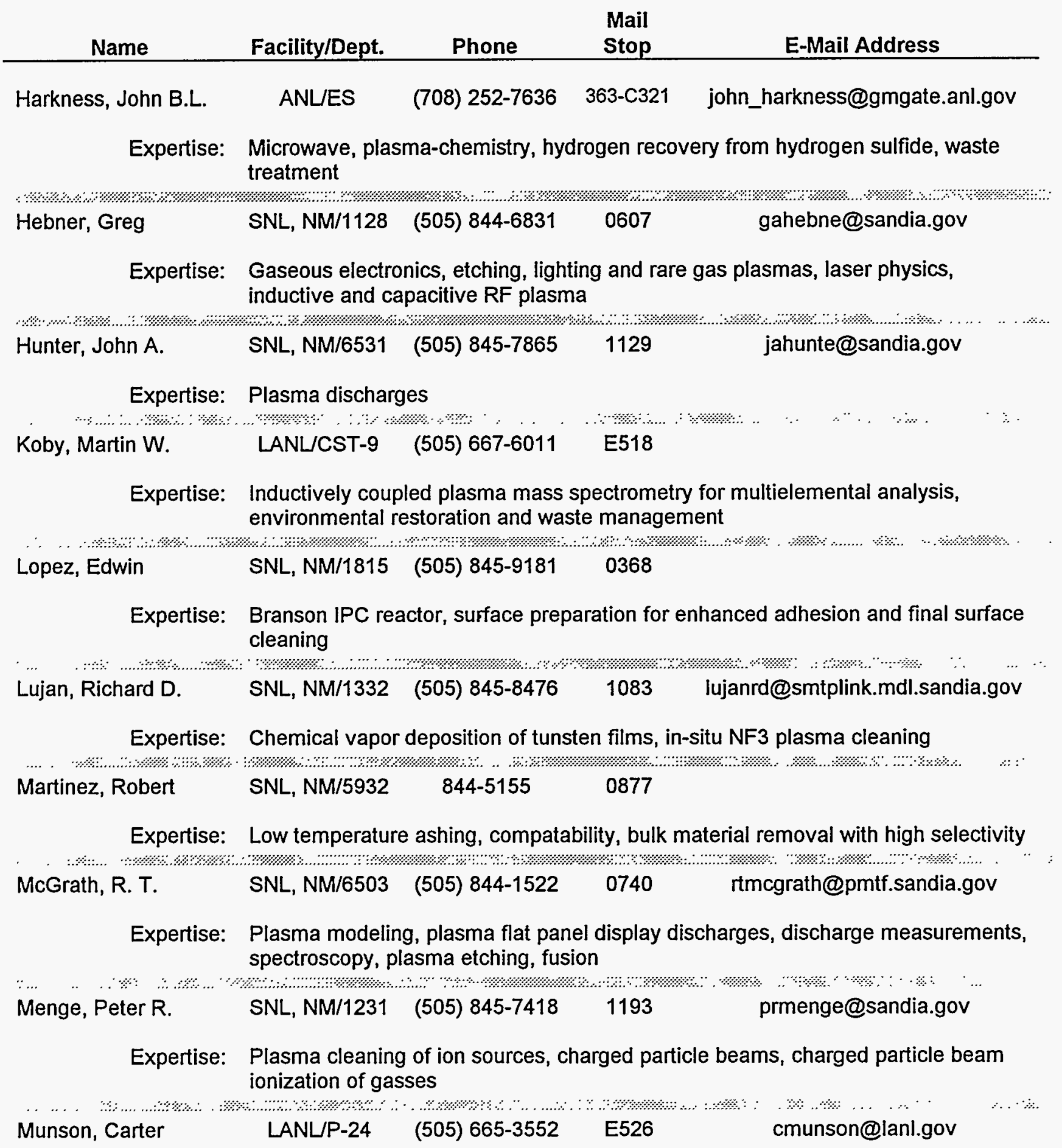

Expertise: Optical, magnetic and particle diagnostics, plasma etching, deposition and implantation, PSII users facility 


\section{Cleaning and Etching continued}

\begin{tabular}{|c|c|c|c|c|}
\hline Name & acility/Dept. & Phone & $\begin{array}{l}\text { Mail } \\
\text { Stop }\end{array}$ & E-Mail Address \\
\hline elson, Patrick W. & NL, NM/1084 & (505) 845-9777 & & pwnelson@smtplink.mdl.san \\
\hline Expertise: & \multicolumn{4}{|c|}{$\begin{array}{l}\text { RIE and plasma etching of silicon nitrides, titanium nitrides, aluminum alloys, etc., } \\
\text { class-1 cleanrooms }\end{array}$} \\
\hline $\mathrm{Ng}$, Henry & ANL & (708) $252-3992$ & $363-\mathrm{b} 24$ & 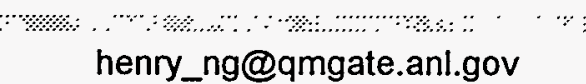 \\
\hline
\end{tabular}

Expertise: Pulsed spark to generate plasma for engines/vehicles to reduce Nox using monatomic nitrogen

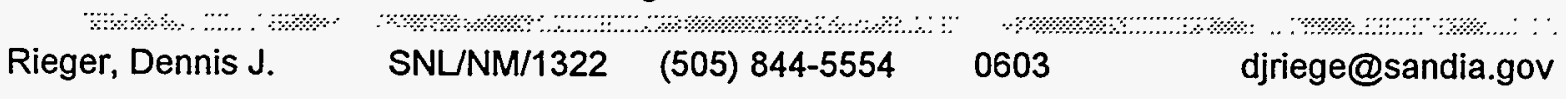

Expertise: Plasma PR stripping, plasma etch and deposition. Hardware installation and evaluation

Robinson, David N. $\quad$ Y-12 (615) $574-2983$

Expertise: Plasma cleaning as a surface treatment for improved adhesion, plasma polymerization for protective coatings

Rosocha, Louis LANLCST-18 (505) 667-8493 E525 rosocha@cst4.lanl.gov

Expertise: Atmospheric pressure nonthermal plasmas, environmental applications, plasma chemistry modeling, chemical processing

Sandoval, Cindy LANLESA-WMA (505) 667-8393 C930 cws@lanl.gov

Expertise: Cleaning, plasma deposition, surface modification

Shul, Randy J. $\quad$ SNL, NM/1322 $\quad(505)$ 844-6126 $0603 \quad$ rjshul@somnet.sandia.gov

Expertise: Barrel asher, RIE, PECVD, ECR, refractory metals, dielectrics, phosphide, arsenic, and nitride-based III-V compound semiconductor

Smith, Mike

SNL, NM/1812 (505) 845-7762 0367

Expertise: Plasma cleaning, etching, deposition, powder production, lasers, optical emission spectroscopy, FTIR, Dektak, etc.

$\begin{array}{llll}\text { Ward, Pamela P. } & \text { SNL, NM/1812 } & (505) 844-2038 & 0367 \quad \text { ppward@sandia.gov }\end{array}$

Expertise: Plasma cleaning, etching, RF inductive and capacitive apparatus, optical emission spectroscopy, interferometry, patterning

Weatherspoon, Kim $\quad \mathrm{Y}-12 \quad$ (615) $576-4228$

Expertise: Plasma cleaning as a surface treatment for improved adhesion, plasma polymerization for protective coatings 


\section{Cleaning and Etching concluded}

\begin{tabular}{ccccc} 
Name & Facility/Dept. & Phone & $\begin{array}{c}\text { Mail } \\
\text { Stop }\end{array}$ & E-Mail Address \\
\hline Wood, Blake P. & LANL/P-24 & $(505) 665-6524$ & E536 & bwood@lanl.gov
\end{tabular}

Expertise: Low-pressure, low-temperature plasma discharge for etching or deposition, cathode arc technology, particle-in-cell models

\section{Deposition}

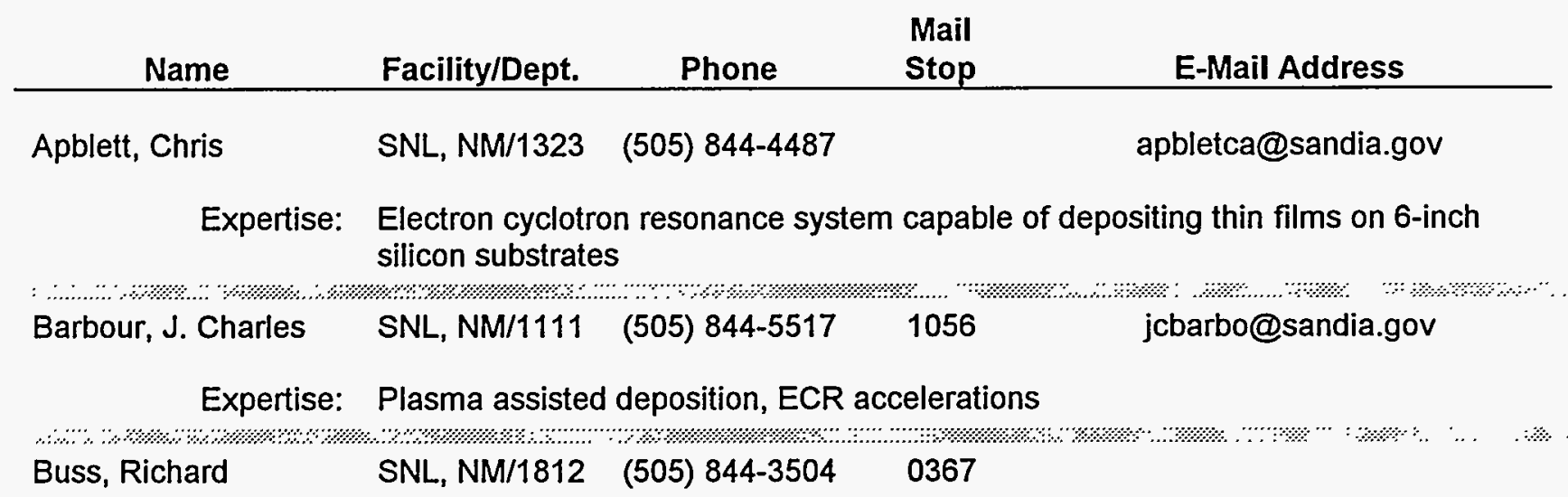

Expertise: Plasma deposition and powder production, optical emission spectroscopy, time-offlight mass spectroscopy, IRIS, ECM

$\begin{array}{lllll}\text { Chang, Chong H. } & \text { INEL } & \text { (208) } 526-2886 & 3808 & \text { chc@inel.gov }\end{array}$

Expertise: Modeling, thermal plasmas, multicomponent, multichemistry, plasma spraying, chemical processing

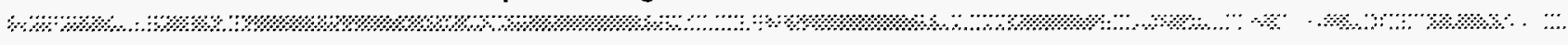

Harkness, John B.L. $\quad$ ANL/ES (708) 252-7636 363-C321 john_harkness@gmgate.anl.gov

Expertise: Microwave, plasma-chemistry, hydrogen recovery from hydrogen sulfide, waste treatment

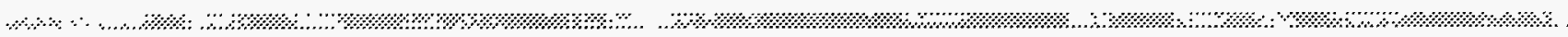

$\begin{array}{llll}\text { Hunter, John A. } & \text { SNL, NM/6531 } & \text { (505) 845-7865 } 1129 \quad \text { jahunte@sandia.gov }\end{array}$

Expertise: Plasma discharges

$\begin{array}{lllll}\text { Krauss, Alan } & \text { ANL } & \text { (708) 252-3520 } & \text { 200-D169 } & \text { alan_krauss@qmgate.anl.gov }\end{array}$

Expertise: Ion beam and plasma-surface interactions, analytical applications of ion beams, i.e., scattering, direct recoil, mass spectroscopy
Lujan, Richard D.
SNL, NM/1332
(505) 845-8476
1083
lujanrd@smtplink.mdl.sandia.gov

Expertise: Chemical vapor deposition of tunsten films, in-situ NF3 plasma cleaning 


\section{Deposition concluded}

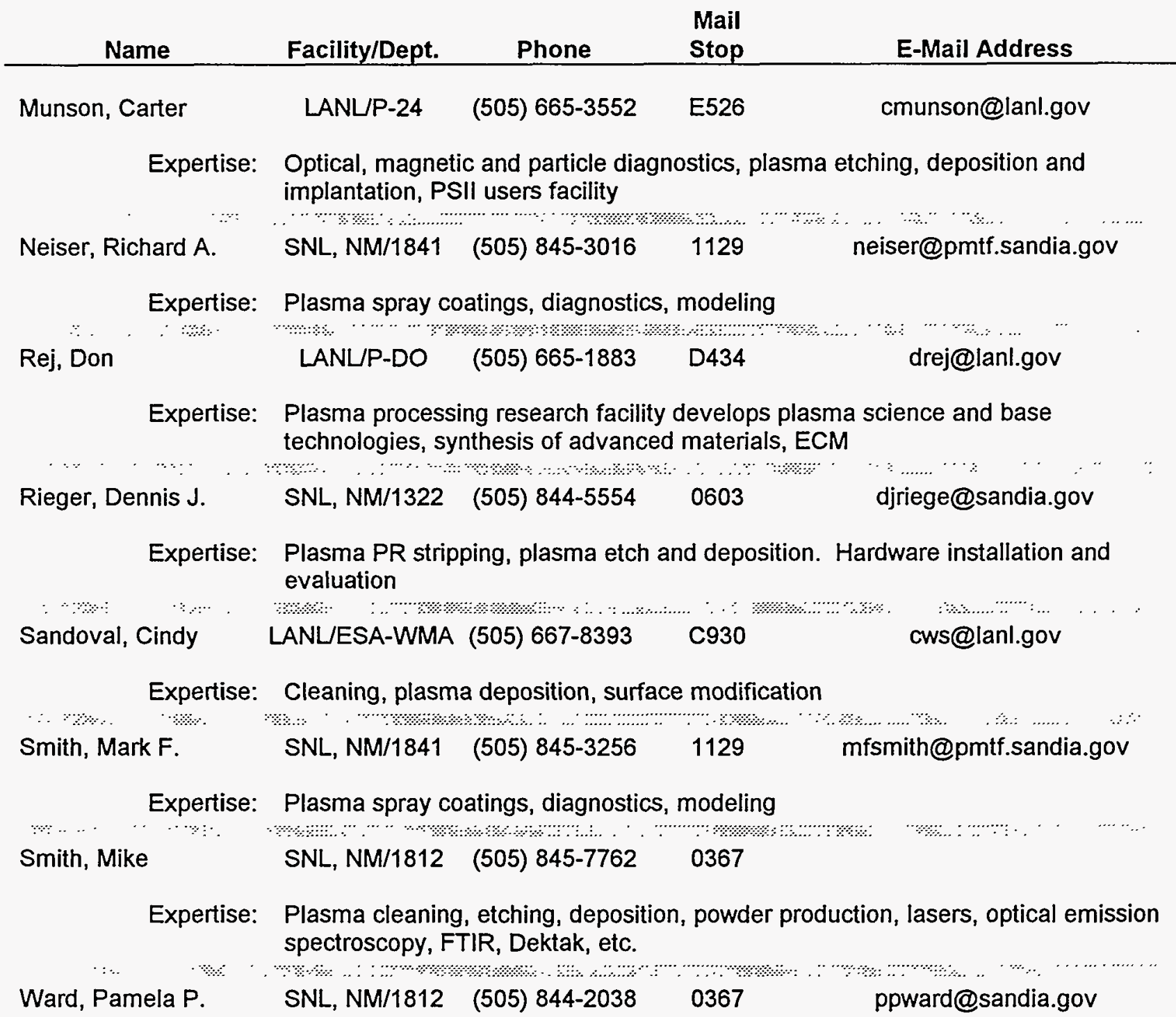

Expertise: Plasma cleaning, RF inductive and capacitive apparatus, optical emission spectroscopy, interferometry, patterning

Wood, Blake P.

$$
\text { LANL/P-24 }
$$$$
\text { (505) 665-6524 }
$$

E536

bwood@lanl.gov

Expertise: Low-pressure, low-temperature plasma discharge for etching or deposition, cathode arc technology, particle-in-cell models
Youchison, Dennis L.
SNL, NM/6531
(505) $845-3138$
1129
dlyouch@sandia.gov

Expertise: ECR chemical vapor deposition, ion implantation 


\section{Diagnostics}

\begin{tabular}{|c|c|c|c|c|}
\hline Name & Facility/Dept. & Phone & $\begin{array}{l}\text { Mail } \\
\text { Stop }\end{array}$ & E-Mail Address \\
\hline Bailey, James E. & SNL, NM/1277 & (505) 845-7203 & 1196 & jebaile@sandia.gov \\
\hline Expertise: & \multicolumn{4}{|c|}{$\begin{array}{l}\text { Z-pinch, laser-produced, and ion diode plasmas, plasma spectroscopy, time and } \\
\text { space resolved spectra }\end{array}$} \\
\hline $\begin{array}{l}\text { B......\%\% } \\
\text { Bastasz, R. }\end{array}$ & SNL, CA/8347 & $(510) 294-2013$ & 9162 & bastasz@vaxm.gat.com \\
\hline
\end{tabular}

Expertise: Plasma-surface interactions, SIMS, AES, ISS

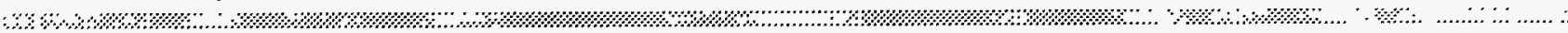

$\begin{array}{lllll}\text { Blackwell, Bennie } & \text { SNL, NM/1514 } & (505) \text { 845-8844 } 0826 \quad \text { blblack@sandia.gov }\end{array}$

Expertise: Modeling of thermal conduction, convection and radiation processes to design and improve plasma equipment

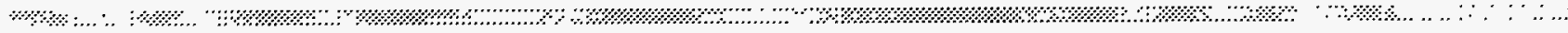

Brockmann, John E. SNL, NM/1512 (505) 844-3201 $0834 \quad$ jebrock@sandia.gov

Expertise: Particle diagnostics

$\begin{array}{lllll} & \\ \text { Buchenauer, D. A. } & \text { SNL, NM/8347 } & \text { (510) 294-3570 } & 9162 & \text { dabuche@ca.sandia.gov }\end{array}$

Expertise: Plasma materials interactions, plasma diagnostics, Languir probes

$\begin{array}{llllll}\text { Buss, Richard } & \text { SNL, NM/1812 } & \text { (505) } 844-3504 & 0367 & & \text { rjbuss@sandia.gov }\end{array}$

Expertise: Plasma deposition and powder production, optical emission spectroscopy, time-offlight mass spectroscopy, IRIS, ECM

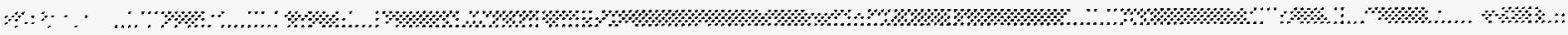
Causey, R. A.
SNL, CA/8347
(510) 294-3326
9162
causey@ca.sandia.gov

Expertise: Plasma-surface interactions, tritium plasma experiment

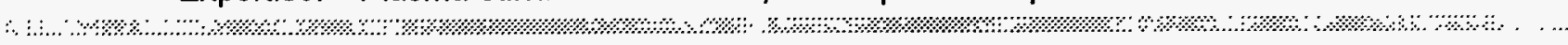
$\begin{array}{lllll}\text { Cobble, James A. } & \text { LANL/P-4 } & \text { (505) 667-8290 E554 cobble@lanl.gov }\end{array}$

Expertise: Spectroscopy, $x$-ray, VUV, laser scattering, fast transient visable and $x$-ray measurements via streak camera, $x$-ray image

$\begin{array}{llll}\text { Cowgill, D. } & \text { SNL, CA/8347 } & \text { (510) 294-2146 } & 9162\end{array}$

Expertise: RF heated plasmas
Dybwad, Gay
SNL, NM/2411 (505) 844-8236
0957
gldybwa@sandia.gov

Expertise: Microwave and downstream plasma etchers, optical emission spectroscopy, circuit cleaning, high rate removal of hydrocarbons, etching of tantalum

$\begin{array}{ccccc}\text { Economou, Demetre } & \text { SNL, NM/1514 } & (505) 844-0124 & 0826 & \text { economou@cfd.sandia.gov }\end{array}$

Expertise: Plasma modeling and diagnostics, multidimensional simulations of plasma reactors, mass spectroscopy, optical emission 


\section{Diagnostics continued}

\begin{tabular}{ccccc} 
Name & Facility/Dept. & Phone & Stop & E-Mail Address \\
\hline Filuk, Alex B. & SNL, NM/1271 & $(505) 845-7385$ & 1187 & abfiluk@sandia.gov \\
Expertise: & $\begin{array}{l}\text { Pulsed-power anode/cathode plasmas, applied-B ion diodes, spectroscopic } \\
\text { diagnosis, spectral line fitting }\end{array}$ & & \\
& LANLNIS-1 & $(505) 665-4314$ & D466 & hfunsten@lanl.gov
\end{tabular}

Expertise: Space plasma instrumentation calibration with ion accelerator and experimental chambers for space flight sensor tests

Gelbard, Fred $\quad$ SNL, NM/1514 (505) 844-5794 $0826 \quad$ fgelbar@cfd.sandia.gov

Expertise: Manager, Equipment Design Support Center, providing total design capabilities for integrated circuit

Haill, Thomas A. SNL, NM/1271 (505) 845-7242 $1187 \quad$ tahaill@sandia.gov

Expertise: Modeling, ion beam transport, ion beam diagnostics

Hareland, Willard A. SNL, NM/1824 (505) 844-7758 0342

Expertise: Langmuir probe, monochromatic imaging, laser light scattering vacuum, ultraviolet spectroscopy

Hassanein, Ahmed

ANL

(708) $252-5889$

207-

hassanein@anlcmt.cmt.anl.gov

EA133A

Expertise: Plasma-material interactions, surface modification effects, design/develop computer codes for plasma-material interactions

Hermina, Wahid

SNL, NM/1514 (505) 844-4759 0826

whemi@sandia.gov

Expertise: Manager, Equipment Design Support Center, providing total design capabilities for integrated circuit

Koby, Martin W.

LANL/CST-9 (505) 667-6011 E518

Expertise: Inductively coupled plasma mass spectrometry for multielemental analysis, environmental restoration and waste management

Krauss, Alan

ANL

(708) 252-3520 200-D169

alan_krauss@qmgate.anl.gov

Expertise: Ion beams and plasma-surface interactions, analytical application of ion beams, I.e., scattering, direct recoil, mass spectroscopy

Lemke, Raymond W.

SNL, NM/1241

(505) $845-7423$

1186

rwlemke@sandia.gov

Expertise: Stability of neutral and nonneutral plasmas, electron beams in gas and vacuum, ion diodes for inertial fusion 


\section{Diagnostics continued}

\begin{tabular}{ccccc} 
Name & Facility/Dept. & Phone & $\begin{array}{c}\text { Mail } \\
\text { Stop }\end{array}$ & E-Mail Address \\
\hline Lutz, Thomas J. & SNL, NM/6531 & $\begin{array}{c}(011) \\
33-9079-5105\end{array}$ & 1129 & lutz@fedv09.cad.cea.fr
\end{tabular}

Expertise: Plasma materials interactions

Mcclellan, George B. SNL,NM/1321 (505) 844-9364 0603

Expertise: Design and modification of plasma equipment, consulting on new designs, modifications, upgrades

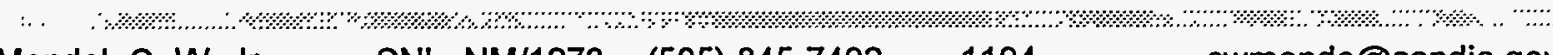

$\begin{array}{lllll}\text { Mendel, C. W. Jr. } & \text { SNL, NM/1273 (505) 845-7492 } 1194 \quad \text { cwmende@sandia.gov }\end{array}$

Expertise: Microwave diagnostics, electrical diagnostics, megampere, megavolt measurements, pulsed plasma sources

$\begin{array}{llll}\text { Munson, Carter } & \text { LANL/P-24 } & (505) 665-3552 & \text { E526 }\end{array}$

Expertise: Optical, magnetic and particle diagnostics, plasma etching, deposition and implantation, PSII users facility

$\begin{array}{lllll}\text { Nash, Tom } & \text { SNL, NM/1273 } & (505) 845-7814 & 0367 & \text { tjnash@sandia.gov }\end{array}$

Expertise: Plasma spectroscopy, plasma imaging, space and nsec time-resolved plasma spectroscopy, $\mathrm{x}$-ray, etc.

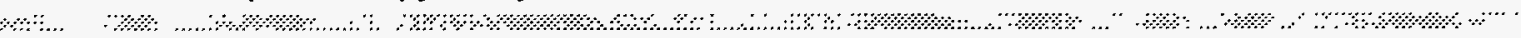

$\begin{array}{llll}\text { Neiser, Richard A. } & \text { SNL, NM/1841 } & (505) \text { 845-3016 } 1129 \quad \text { neiser@pmtf.sandia.gov }\end{array}$

Expertise: Plasma spray coatings, diagnostics, modeling

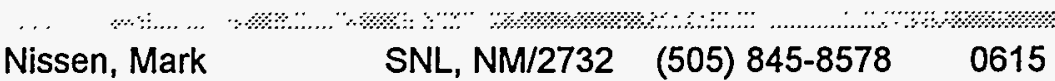

Expertise: Ultra-high-speed photography and spectroscopy and other diagnostics of plasma phenomenology

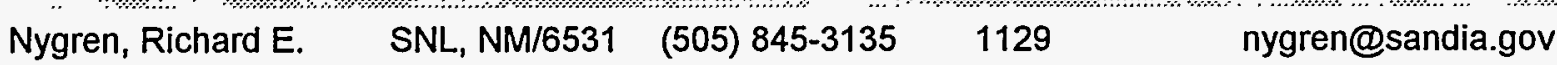

Expertise: Plasma materials interactions

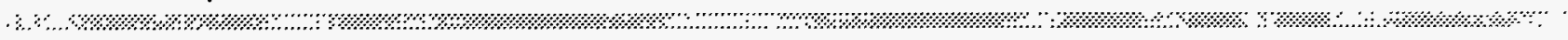

$\begin{array}{llll}\text { O'Hern, Timothy } & \text { SNL, NM/1512 (505) 844-9061 } 0834 \quad \text { thohern@sandia.gov }\end{array}$

Expertise: Particle diagnostics

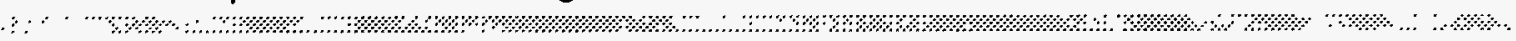

$\begin{array}{lllll}\text { Pointon, Timothy D. } & \text { SNL, NM/1242 (505) 845-7641 } 1193 \quad \text { tdpoint@sandia.gov }\end{array}$

Expertise: Particle-in-cell computer modeling of plasmas, large-scale 2- and 3-D

electromagnetic PIC simulations 


\section{Diagnostics continued}

\begin{tabular}{rcccc} 
Name & Facility/Dept. & Phone & $\begin{array}{c}\text { Mail } \\
\text { Stop }\end{array}$ & E-Mail Address \\
\hline Rej, Don & LANL/P-DO & $(505) 665-1883$ & D434 & drej@lanl.gov
\end{tabular}

Expertise: Plasma processing research facility develops plasma science and base technologies, synthesis of advanced materials, ECM

$\begin{array}{llll}\text { Rockett, Paul D. } & \text { SNL, NM/6531 } & \text { (505) 845-7466 } 1129 & \text { pdrocke@sandia.gov }\end{array}$

Expertise: High-density plasma, magnetic fusion disruption simulation, x-ray, EUV, UV spectroscopy

Russo, Anthony J. SNL, NM/6531 (301) 903-6288 1129 anthony.russo@mailgw.er.doe.gov

Expertise: Runaway electron generation

Sanford, T. W. L. $\quad$ SNL, NM/1231 (505) 845-7816

Expertise: Electron diodes, intense electron beam transport, Hermes III, calorimeters, fast framing $x$-ray camera

$\begin{array}{llll}\text { Selwyn, Gary S. } & \text { LANL/P-24 } & \text { (505) 665-7359 E526 } & \text { gas@fjwsys.lanl.gov }\end{array}$

Expertise: Real-time, in-situ detection and control of particulate contamination, design modification for process uniformity

Shagan, Richard

SNL, NM/2732 (505) 845-9079 0615

Expertise: Ultra-high-speed photography and spectroscopy and other diagnostics of plasma phenomenology

Smith, David Lewis SNL, NM/1239 (505) 845-7141 1184

Expertise: High-voltage, high-current gas switching, high-current electron beam diode development, laser triggered

Smith, Mark F.

SNL, NM/1841 (505) 845-3256 1129

mfsmith@pmtf.sandia.gov

Expertise: Plasma spray coatings, diagnostics, modeling

Smith, Mike

SNL, NM/1812 (505) 845-7762 0367

Expertise: Plasma cleaning, etching, deposition, powder production, lasers, optical emission spectroscopy, FTIR, Dektak, etc.

Stevens, J. E.

SNL, NM/1326 (505) 844-2802

1077

James_E._Stevens@smtplink.mdl.sandia.gov

Expertise: Plasma and RF diagnostics, RFI/ICP, ECR, helicon, diode and microwave plasma reactor 
Diagnostics concluded

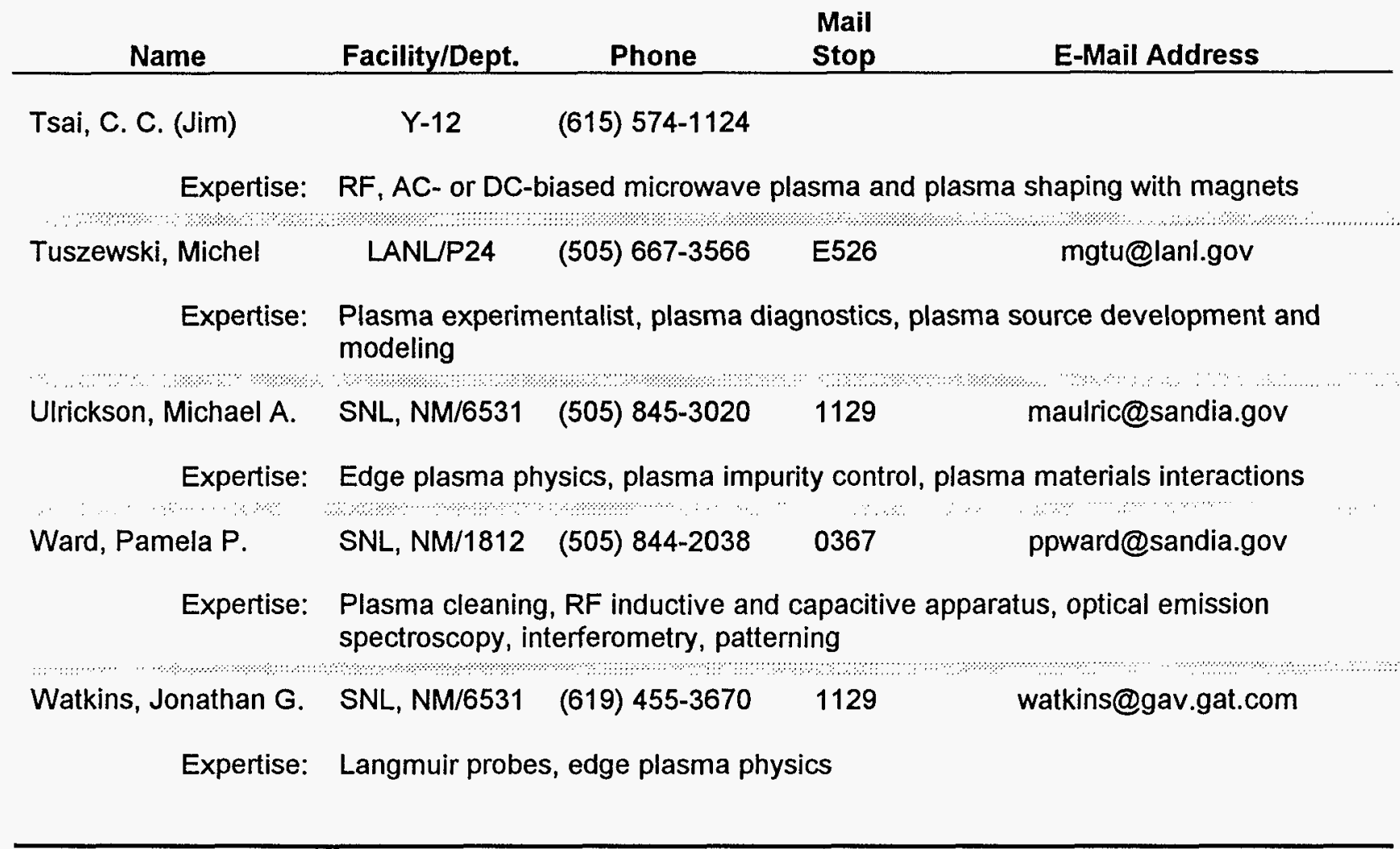

\section{Modeling}

\begin{tabular}{|c|c|c|c|c|}
\hline Name & Facility/Dept. & Phone & $\begin{array}{l}\text { Mail } \\
\text { Stop }\end{array}$ & E-Mail Address \\
\hline Bartel, Tim & SNL, NM/1514 & (505) 844-0124 & 0826 & tjbarte@cfd.sandia.gov \\
\hline Expertise: & \multicolumn{4}{|c|}{$\begin{array}{l}\text { Numerical simulation of plasma etch and deposition reactors and processes } b \\
\text { self-consistent particle method }\end{array}$} \\
\hline Blackwell, Bennie & SNL, NM/1514 & (505) $845-8844$ & 0826 & blblack@sandia.gov \\
\hline Expertise: & \multicolumn{4}{|c|}{$\begin{array}{l}\text { Modeling of thermal conduction, convection and radiation processes to design } \\
\text { improve plasma equipment }\end{array}$} \\
\hline Campbell, R. B. & SNL, NM/1512 & (505) 844-6205 & 0834 & rbcampb@sandia.gov \\
\hline Expertise: & \multicolumn{4}{|c|}{$\begin{array}{l}\text { Models neutral gas flow, particle transport, fully ionized magnetized plasma, } \\
\text { microwave propagation, plasma modeling, plasma display discharges, fusion } \\
\text { plasmas }\end{array}$} \\
\hline
\end{tabular}




\section{Modeling (continued)}

\begin{tabular}{ccccc} 
Name & Facility/Dept. & Phone & $\begin{array}{c}\text { Mail } \\
\text { Stop }\end{array}$ & E-Mail Address \\
\hline Chang, Chong H. & INEL & $(208) 526-2886$ & 3808 & chc@inel.gov
\end{tabular}

Expertise: Modeling, thermal plasmas, multicomponent, multichemistry, plasma spraying, chemical processing

Choi, Seung J. .

$0834 \quad$ sjchoi@engsci.sandia.gov

Expertise: Plasma chemistry, modeling, gas phase particulate contamination

$\begin{array}{llll}\text { Doyle, Barney L. } & \text { SNL, NM/1111 } & \text { (505) 844-7568 } 1056 \quad \text { bldoyle@sandia.gov }\end{array}$

Expertise: Plasma surface interactions, ion sources accelerators

Dykhuizen, R.C. SNL, NM/1513 $\quad$ (505) $844-910500350$ rcdykhu@sandia.gov

Expertise: Analytical modeling

Economou, Demetre SNL, NM/1514 (505) 844-0124 $0826 \quad$ economou@cfd.sandia.gov

Expertise: Plasma modeling and diagnostics, multidimensionall simulations of plasma reactors, mass spectroscopy, optical emission

$\begin{array}{lllll}\text { Haill, Thomas A. } & \text { SNL, NM/1271 } & \text { (505) 845-7242 } 1187 & \text { tahaill@sandia.gov }\end{array}$

Expertise: Modeling, ion beam transport, ion beam diagnostics

Hassanein, Ahmed

ANL

(708) 252-5889 207-

hassanein@anlcmt.cmt.anl.gov EA133A

Expertise: Plasma-material interaction, surface modification effects, design/develop computer codes for plasma-material interactions

Johannes, Justine SNL, NM/1514 (505) 844-1994 $0826 \quad$ jejohan@cfd.sandia.gov

Expertise: Numerical simulation of plasma etch and deposition chemistry processes using particle simulation code

$\begin{array}{llll}\text { Kanouff, Michael P. SNL, CA/9043 } & (510) 294-2105 \quad 8743 \quad \text { kanouff@california }\end{array}$

Expertise: Particle impact and surface modeling

McClellan, George B. SNL, NM/1321 (505) 844-9364 0603

Expertise: Design and modification of plasma equipment, consulting on new designs, modifications, upgrades

$\begin{array}{lllll}\text { McGrath, R. T. } & \text { SNL, NM/6503 (505) 844-1522 } 0740 \quad \text { rtmcgrath@pmtf.sandia.gov }\end{array}$

Expertise: Plasma modeling, plasma flat panel display discharges, discharge measurements, spectroscopy, plasma etching, fusion 
Modeling (continued)

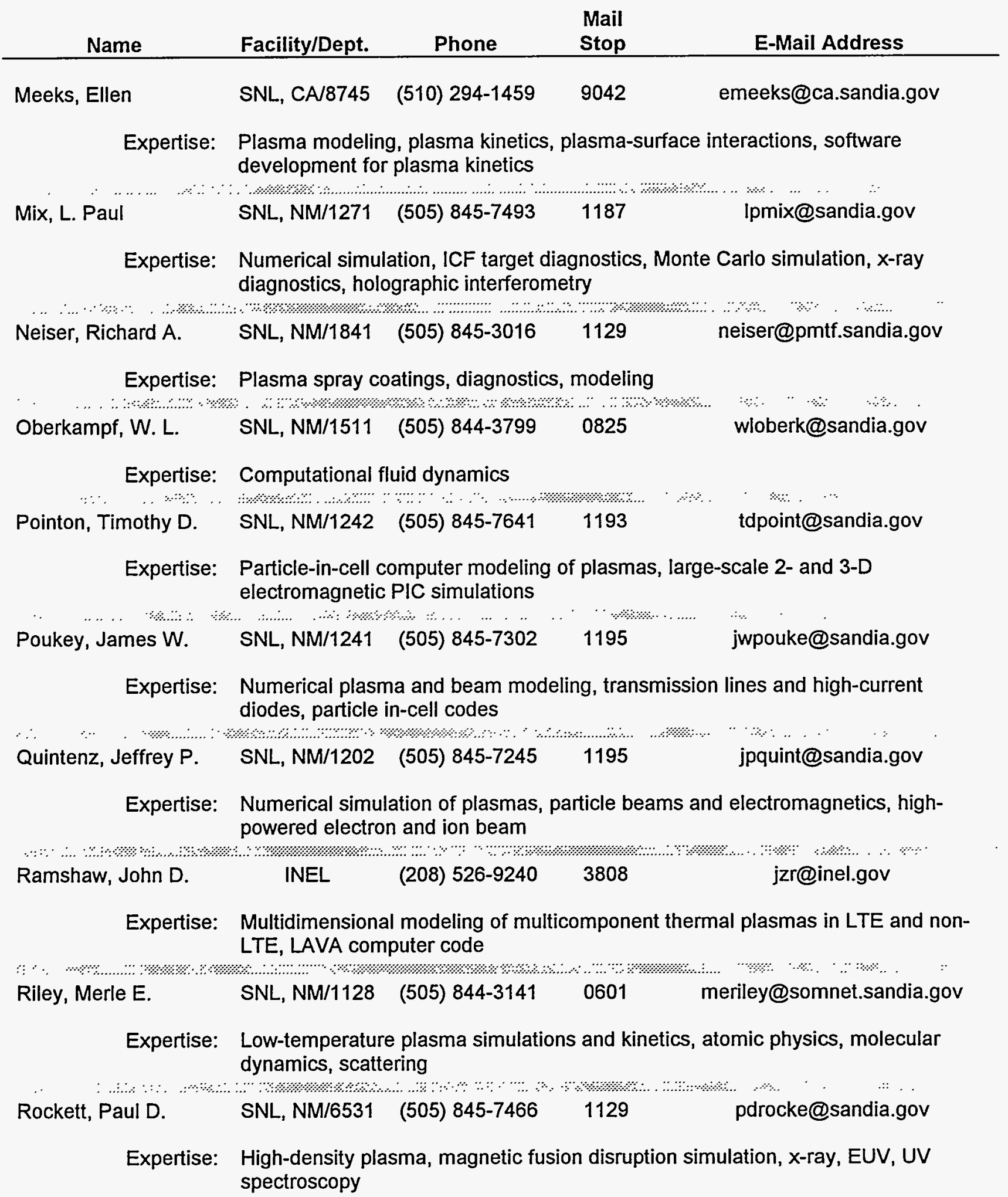


Modeling (concluded)

\begin{tabular}{|c|c|c|c|c|}
\hline Name & Facility/Dept. & Phone & $\begin{array}{l}\text { Mail } \\
\text { Stop }\end{array}$ & E-Mail Address \\
\hline Rosocha, Louis & LANL/CST-18 & (505) 667-8493 & E525 & rosocha@cst4.lanl.gov \\
\hline Expertise: & \multicolumn{4}{|c|}{$\begin{array}{l}\text { Atmospheric pressure nonthermal plasmas, environmental applications, plasma } \\
\text { chemistry modeling, chemical processing }\end{array}$} \\
\hline Russo, Anthony J. & SNL, NM/6531 & (301) 903-6288 & 1129 & anthony.russo@mailgw.er.doe.gov \\
\hline Expertise: & \multicolumn{4}{|c|}{ Runaway electron generation } \\
\hline Smith, Mark F. & SNL, NM/1841 & (505) $845-3130$ & 1129 & mfsmith@pmtf.sandia.gov \\
\hline $\begin{array}{l}\text { Expertise: } \\
\text { Tuszewski, Michel }\end{array}$ & $\begin{array}{c}\text { Plasma spray c } \\
\text { LANL/P24 }\end{array}$ & $\begin{array}{r}\text { atings, diagnostic } \\
\text { (505) 667-3566 }\end{array}$ & $\begin{array}{l}\text { modelir } \\
\text { E526 }\end{array}$ & mgtu@lanl.gov \\
\hline Expertise: & \multicolumn{4}{|c|}{$\begin{array}{l}\text { Plasma experimentalist, plasma diagnostics, plasma source development and } \\
\text { modeling }\end{array}$} \\
\hline Veerasingam, $R$. & SNL, NM/6531 & (505) $845-3490$ & & ramana@pmtf.sandia.gov \\
\hline
\end{tabular}

Walsh, David S. $\quad$ SNL, NM/1111 (505) 844-9590 $1056 \quad$ dswalsh@sandia.gov

Expertise: Plasma surface interactions, ion sources accelerators

$\begin{array}{lllll}\text { Wampler, William R. SNL, NM/1111 } & \text { (505) 844-4114 } & 1056 \quad \cdots & \text { wrwampl@sandia.gov }\end{array}$

Expertise: Plasma surface interactions, ion sources accelerators

Wood, Blake P. $\quad$ LANL/P-24 (505) 665-6524 E536 bwood@lanl.gov

Expertise: Low-pressure, low-temperature plasma discharge for etching or deposition, cathode arc technology, particle-in-cell models 


\section{Address/Change Form}

If you would like to be included in the the next edition of the Plasma Technology Directory, or if you would like to change or correct your current listings, please complete this form and return it to:

P. Ward, SNL, NM, MS 0367

ppward@sandia.gov

(505) 844-2038

(505) 844-9624 fax

Name

Facility

Address

(include department number and

mail stop)

Telephone

E-Mail Address

Area of expertise: 
Distribution:

10 DOE laboratories Technology Transfer groups

All Directory participants

1 MS 9018 Central Technical Files, 8523-2

5 MS 0899 Technical Library, 13414

$1 \quad$ MS 0619 Print Media, 12615

2 MS 0100 Document Processing, 7613-2 for DOE/OSTI 